\title{
1 \\ Modelling the population size and dynamics of the British grey seal
}

2 Len Thomas ${ }^{1}$, Debbie J.F. Russell ${ }^{1,2}$, Callan Duck ${ }^{2}$, Chris D. Morris ${ }^{2}$, Mike Lonergan $^{2,3}$, Fanny Empacher ${ }^{1}$,

3 Dave Thompson ${ }^{2}$ and John Harwood ${ }^{1}$.

$4{ }^{1}$ Centre for Research into Ecological and Environmental Modelling, Scottish Oceans Institute, $5 \quad$ University of St Andrews, Scotland.

6

${ }^{2}$ Sea Mammal Research Unit, Scottish Oceans Institute, University of St Andrews, Scotland.

7

${ }^{3}$ Medical Research Institute, University of Dundee, Dundee, Scotland.

8

9

Correspondence: Len Thomas, Centre for Research into Ecological Environmental Modelling, The Observatory, Buchanan Gardens, University of St Andrews, St Andrews, Fife, KY16 9LZ, Scotland. len.thomas@st-andrews.ac.uk

\section{Abstract}

1. Grey seals (Halichoerus grypus Fabricius, 1791) were the first mammals to be protected by an Act of Parliament in the UK and are currently protected under UK, Scottish and EU conservation legislation. Reporting requirements under each of these statutes requires accurate and timely population estimates. Monitoring is principally conducted by aerial surveys of the breeding colonies; these are used to produce estimates of annual pup production. Translating these to estimates of adult population size requires information about demographic parameters such as fecundity and sex ratio.

2. An age-structured population dynamics model is presented, which includes density dependence in pup survival, with separate carrying capacities in each of the four considered breeding regions (North Sea, Inner Hebrides, Outer Hebrides, and Orkney). This model is embedded within a Bayesian state-space modelling framework, allowing the population 
model to be linked to available data and the use of informative prior distributions on demographic parameters. A computer-intensive fitting algorithm is presented based on particle filtering methods.

3. The model is fitted to region-level pup production estimates from 1984-2010 and an independent estimate of adult population size derived from aerial surveys of hauled-out seals in 2008. The fitted model is used to estimate total population size from 1984-2010.

4. The population in the North Sea region has increased at a near-constant rate; growth in the other three regions began to slow in the mid-1990s and these populations appear to have reached carrying capacity. Total population size of age 1+ seals in 2010 was estimated to be 116,100 (95\% Cl 98,400-138,600), an increase of $<1 \%$ on the previous year.

5. The modelling and fitting methods are widely applicable to other wildlife populations where diverse sources of information are available and inference is required about the underlying population dynamics.

Keywords: Bayesian statistics, delayed density dependence, Halichoerus grypus, integrated population monitoring, particle filter, population trend, population dynamics, sequential Monte Carlo, state-space model. 


\section{Introduction}

Obtaining reliable estimates of population size and trend is a key goal of many wildlife monitoring programmes. In many cases, population size can be estimated for each monitoring period (e.g. annually) using survey methods such as distance sampling or mark-recapture (Borchers, Buckland, \& Zucchini, 2002). Population trend can then be inferred by smoothing the resulting time series (Thomas, Burnham, \& Buckland, 2004). However, in some situations, only a component of the population can be readily surveyed - for example breeding females or new-born offspring. Scaling up to total population size then requires knowledge of what proportion of the population is represented by the component surveyed. Partial information relevant to the required proportion may be available from multiple sources. One way to bring all this information together in an integrated way is to embed it within a statistical model of population dynamics (Newman et al., 2014). This has the additional advantage that the resulting time series of population estimates is constrained to be biologically realistic (unlike purely empirical smooths of population trend) so may be useful for trend estimation even when the entire population can be surveyed. Furthermore, population dynamics modelling helps determine which particular processes influence population size and changes

In this paper, Bayesian statistical techniques are used to fit an age-structured model of British grey seal population dynamics to annual estimates of pup production (number of pups born per year), a single estimate of total population size and diverse data on demographic parameters in the form of informative prior distributions. This model is used to infer population size and trend over multiple years as well as to examine region-level density dependence.

The British population of grey seals (Halichoerus grypus Fabricius, 1791) makes up approximately 40\% of the world population (SCOS, 2017). It was historically much reduced by commercial and subsistence hunting, and concern about possible extirpation led in 1914 to it becoming the first mammal in the UK to be protected by an act of Parliament (Lambert, 2002). Subsequent legislation (The UK Conservation of Seals Act 1970 and The Marine (Scotland) Act 2010) placed a statutory requirement on the Natural 
Environment Research Council (NERC) to provide scientific advice to UK and Scottish governments on matters related to the management of seal populations. NERC created an expert panel, the Special Committee on Seals (SCOS), to provide this advice, informed by scientific information from the Sea Mammal Research Unit (SMRU). This information includes assessments of population size and trend, derived using the methods described here. A historical example of advice given around a highly contentious topic relates to the extent of competition between grey seals and fisheries, and the utility of large-scale culls (reviewed by Harwood \& Greenwood 1985). A more recent example is an investigation of the potential role of grey seals in regional harbour seal population declines (Thompson, Duck, Morris, \& Russell, Submitted).

Grey seals are a good example of a species in which only one component of the population is readily surveyed; they spend most of the year at sea on foraging trips, widely dispersed at low density, but congregate annually at a small number of breeding colonies to pup, and pups remain ashore for approximately the first month of life (Wyile, 1988). Population monitoring has, therefore, focused on counting pups, either from the ground or from aerial photographic surveys. The breeding season is longer than the maximum length of stay for any individual pup, and hence no single survey will count all pups born at a colony. Repeated counts are therefore required, and a statistical model is used to estimate total pup production per colony from the count data (Russell, Morris, Duck, Thompson, and Hiby, Submitted).

Pup counts have taken place in the UK since the 1950s, and an assessment of regional trends in pup production, showing an approximately exponential increase of $7 \%$ per year in areas undisturbed by culling, was published by SMRU in the year of its founding (Summers, 1978). Under constant population growth (assuming constant age-specific survival and fecundity) total population size is a simple multiple of pup production, with the value of the multiplier depending on population age structure and age-specific fecundity. A companion paper (Harwood \& Prime, 1978) used the age distribution of a sample of culled adult seals, and other information, to infer that the appropriate 
multiplier corresponding to a growth rate of 1.07 is approximately 4 (their Figure 6 ). This paper also considered how a density-dependent decrease in population growth might affect this multiplier and found that the effect depended on which demographic parameter was affected by density dependence. They suggested that the most likely parameter would be pup survival, and that a decrease in pup survival sufficient to cause a levelling-off of pup production would result in the multiplier decreasing from 4 to 3 . This current paper, appearing in a special issue celebrating the $40^{\text {th }}$ anniversary of SMRU (Hall, this issue), can be seen as an update to the Harwood and Prime paper, using a new approach to model the population. An earlier version of the model and fitting algorithm used here was described in Thomas, Buckland, Newman, and Harwood (2005) and Newman, Buckland, Lindley, Thomas, and Fernández (2009), so this paper is also an update to those.

Although the focus of this paper is on British grey seals, the methods used are general, readily modified to other age- or stage-structured wildlife populations. Details of the fitting algorithm, and computer code, are provided as Supporting Information.

\section{Methods}

\subsection{Pup production estimates}

Estimates of pup production have been calculated for some colonies since the 1950s (see Figure 6 and Supplementary Material in Russell et al. Submitted), but initial counts did not cover all major colonies and methods for counting and estimating pup production have changed over time. In this paper, only annual pup production estimates for 1984-2010 were used (Table S1, Supporting Information); aerial survey methods changed substantially after 2010 . The pup production estimates used are those derived by Russell et al. (Submitted). Pup production can fluctuate widely over time within a colony, and nearby colonies can show divergent trends. Hence, to facilitate population modelling, colony-level pup production estimates were aggregated into four study regions: Inner Hebrides (which, in terms of grey seal colonies, is synonymous with the West Scotland Seal Management Unit (SMU)), Outer 

value: in 2009 there were too few aerial surveys in Inner Hebrides for a reliable pup production estimate to be produced.

Approximately $10 \%$ of breeding occurs in colonies not regularly surveyed or outside of these regions. Hence models fitted to the pup production data only estimate the population size of seals associated with regularly monitored colonies.

The statistical procedure used to estimate pup production from aerial surveys at the colony level yields an associated measure of uncertainty. However, several parameters involved in the estimation are shared among colonies within regions and hence colony-level estimates are not independent, making it difficult to produce an aggregate estimate of region-level uncertainty. In addition, no measure of uncertainty is available for pup production estimates made from ground counts. Hence, the level of observation error associated with the regional pup production estimates is estimated in the population dynamics modelling process (Section 2.4) rather than being assumed known.

\subsection{Independent estimate of population size}

Grey seals also haul out of the water during the non-breeding season between foraging trips. Although these haul-out sites are much more widely dispersed than the breeding sites, it is possible to survey them using aerial photography. This was conducted primarily within a 3-year period 2007-2009 as part of a larger survey (of harbour seals) during August, within 2 hours of local low tide (Lonergan, Duck, Thompson, Moss, \& McConnell, 2011). The data collected were used to derive an independent estimate of total population size, nominally assigned to 2008 , by dividing the total seals hauled out by the proportion of the population estimated to be hauled out during the survey. This latter proportion was estimated separately using a sample of seals fitted with telemetry tags. Here, the estimate 
derived by Russell, Duck, Morris, and Thompson (2016) was used, which is an update of Lonergan et al. (2011).

The population dynamics model fitted to pup production data produces estimates of total population size for only the regularly-monitored colonies. To account for this, the estimate from Russell et al. (2016) was scaled downwards by multiplying it by 0.9234 , which is the proportion of pup production estimated to have taken place on regularly-monitored colonies in 2008 (Duck, 2009). This assumes that the number of adults per pup is the same within and outside the regularly-monitored colonies.

Russell et al. (2016) used a non-parametric bootstrap to quantify uncertainty on the total population size estimate. The method used here to link the total population size estimate to the pup production model requires the former to be expressed as a parametric distribution. To allow this, the bootstrap replicates from Russell et al. (2016), after scaling, were modelled as arising from a right-shifted gamma distribution

$$
Y_{200} \widetilde{8}_{0}+\mathrm{Ga}\left(\kappa_{1}, \kappa_{2}\right)
$$

where $Y_{20} 0$ dis the estimated total population size in 2008 after scaling, $\sim$ denotes "is distributed according to", $\kappa_{0}$ is the right shift (non-negative), and $\mathrm{Ga}\left(\kappa_{1}, \kappa_{2}\right)$ denotes a gamma distribution with shape parameter $\kappa_{1}$ and scale parameter $\kappa_{2}$. (Note that, more strictly, we should write $Y_{20} 08$ $\kappa_{0} \sim \operatorname{Gamma}\left(\kappa_{1}, \kappa_{2}\right)$ but the above informal notation is used throughout this paper for convenience.) The parameters $\left\{\kappa_{0}, \kappa_{1}, \kappa_{2}\right\}$ were estimated by maximum likelihood from 100,000 bootstrap replicates.

\subsection{Population dynamics model}

A discrete time, age-structured stochastic population dynamics model was constructed for each region, with most demographic parameters shared across regions. Female seals recruit into the breeding population at around 6 years old (Harwood and Prime 1978). In this model, the seal population in each region is divided into seven age classes: pups (age 0), pre-breeding females in age 
classes 1 to 5 and breeding-age females (age 6 and older). Note that all animals age 1 or older are referred to as "adult", although in practice those age 1-5 could more accurately be described as immature or sub-adult. Note also that the population dynamics model does not explicitly include adult males - see Section 2.4. The time step is 1 year, beginning just after the breeding season. The number of seals of age $\mathrm{x}$ in region $\mathrm{r}$ at time $\mathrm{t}$ is denoted as $n_{x, r, t}$. The year is divided into three sub-processes, occurring sequentially: survival, age incrementation, and breeding.

Survival within each region and age class is modelled as a binomial random process, with different survival probability for pups and adults:

$$
\begin{aligned}
& u_{\mathrm{s}, 0, r, t} \sim \operatorname{Bi}\left(n_{0, r, t-1}, \phi_{p, r, t}\right) \\
& u_{\mathrm{s}, x, r, t} \sim \operatorname{Bi}\left(n_{x, r, t-1}, \phi_{a}\right) \quad x=1, \ldots 6,
\end{aligned}
$$

where $u_{\mathrm{s}, x, r, t}$ is the number of seals age $x$ in region $r$ at time $t$ after the survival sub-process but before age incrementation; $\phi_{p, r, t}$ is the survival probability of pups in region $r$ at time $t$; and $\phi_{a}$ is the survival probability of adults (assumed constant across regions and time). Following Harwood and Prime (1978), pup survival is assumed to be density dependent, i.e. to decline as the number of pups increases. The density dependent function used here is

$$
\phi_{p, r, t}=\frac{\phi_{\mathrm{pmax}}}{1+\left(\beta_{\mathrm{r}} \mathrm{n}_{0, \mathrm{r}, \mathrm{t}-1}\right)^{\rho}}
$$

where $\phi_{p \max }$ is maximum pup survival, attained when pup production is zero, and $\beta_{r}$ and $\rho$ determine how pup survival declines as pup numbers increase. The parameter $\beta_{r}$ controls the overall rate of the decrease, while $\rho$ controls the shape of the function (Figure S2, Supporting Information). When $\rho=1$ Eqn. (1) is the classic Beverton-Holt function (Beverton \& Holt, 1957), while increasing $\rho$ above 1 causes pup survival to remain close to the maximum at low levels of pup production and decrease rapidly at higher pup production. Including a density dependent demographic parameter in the model means that the rate of population growth will slow as the population increases, and the population will stabilize at (or oscillate around) a fixed population size (the carrying capacity) and age structure 
184 (Caswell 2001, Turchin 2003). Carrying capacity is a function of all the demographic parameters in the model; however, because the only parameter that varies between regions is $\beta_{r}$, this is the parameter that controls the relative size of the carrying capacity in each region.

For age incrementation, the number of surviving pups that become age 1 females is a binomial random variable with probability 0.5 , since (by assumption) on average only half of the pups will be male. For other age classes, age incrementation is deterministic, with all seals ageing by one year, except those in the $6+$ category which remain in that age class. This part of the model can be summarized as

$$
\begin{aligned}
& u_{\mathrm{a}, 1, r, t} \sim \operatorname{Bi}\left(u_{\mathrm{s}, 0, r, t}, 0.5\right) \\
& u_{\mathrm{a}, x, r, t}=u_{\mathrm{s}, x-1, r, t} \\
& u_{\mathrm{a}, 6, r, t}=u_{\mathrm{s}, 5, r, t}+u_{\mathrm{s}, 6, r, t}
\end{aligned} \quad x=2, \ldots, 5
$$

where $u_{\mathrm{a}, x, r, t}$ is the number of seals age $x$ in region $r$ at time $t$ after the age incrementation sub-process but before breeding.

194 To model breeding, it is assumed that each age 6 and older female gives birth to a single pup with probability $\alpha$, so that the number of pups is a binomial random variable:

$$
n_{0, r, t} \sim \operatorname{Bi}\left(u_{a, 6, r, t}, \alpha\right)
$$

197 One way to summarize the model is using matrix notation (Caswell 2001), showing the expected number of seals at time $t+1$ :

$$
\mathrm{E}\left(\boldsymbol{n}_{r, t+1}\right)=\boldsymbol{L} \boldsymbol{n}_{r, t}
$$
in region $r$ at time $t, E($.$) is the expectation operator, and \boldsymbol{L}$ is a Leslie matrix, which for this model is given by 


$\boldsymbol{L}=\begin{array}{ccccccc}0 & 0 & 0 & 0 & 0 & \phi_{a} \alpha & \phi_{a} \alpha \\ 0.5 \phi_{p, r, t} & 0 & 0 & 0 & 0 & 0 & 0 \\ 0 & \phi_{a} & 0 & 0 & 0 & 0 & 0 \\ 0 & 0 & \phi_{a} & 0 & 0 & 0 & 0 \\ 0 & 0 & 0 & \phi_{a} & 0 & 0 & 0 \\ 0 & 0 & 0 & 0 & \phi_{a} & 0 & 0 \\ 0 & 0 & 0 & 0 & 0 & \phi_{a} & \phi_{a}\end{array}$

204

205

206

207

208

209

210

211

212

213

214

215

216

217

218

\subsection{State-space model}

A convenient way to link the above population dynamics model to observations made on its components is using a state-space modelling framework (Newman et al., 2014). A state-space model describes the evolution in time of two linked stochastic processes: a state process and an observation process. The state process describes the true but unknown ("hidden") state of the population at successive time steps - in this case this is given by the population dynamics model described above. The observation process links the unknown states (the number of animals in each age class in each region) to data on the populations.

To link pup production to the pup production estimates it was assumed that the pup production estimates for each region and year are normally distributed with a constant (but unknown) coefficient of variation:

$$
y_{0, r, t} \sim \mathrm{N}\left(\mathrm{n}_{0, \mathrm{r}, \mathrm{t}} \frac{\mathrm{n}_{0, \mathrm{r}, \mathrm{t}}^{2}}{\psi}\right)
$$

where $y_{0, r, t}$ is the pup production estimate for region $r$ and year $t, \mathrm{~N}\left(\mu, \sigma^{2}\right)$ denotes a normal distribution with mean $\mu$ and variance $\sigma^{2}$, and $\psi$ is a precision parameter, so that the coefficient of variation of the pup production estimate is $1 / \sqrt{\psi}$.

To link total population size to the independent estimate made in 2008 , it was assumed that the independent estimate followed a right-shifted gamma distribution with known parameters $\left\{\kappa_{0}, \kappa_{1}, \kappa_{2}\right\}$ (see Section 2.2). The mean of this distribution, $\kappa_{0}+\kappa_{1} \kappa_{2}$, was assumed equal to the true total population size of adults (on regularly monitored colonies) in 2008, $N_{20} 0.8$ Only adults are included 
223 (i.e. pups are excluded) because the independent estimate took place in August, i.e. before the breeding season. (It was assumed that adult mortality between the time of the independent estimate and the end of the breeding season, which is the population model census point, was negligible.) The population dynamics model includes only adult females, so an additional parameter, $\omega$, is required to index the number of adult animals per adult female:

$$
N_{200}=\omega \sum_{x=1}^{6} \sum_{r=1}^{4} n_{x, r, 2008}
$$

The parameter $\omega$ is related to the adult sex ratio, in that the ratio of adult males to adult females is given by $\omega \quad 1$.

One further step is required to fully specify the state-space model: defining the distribution of initial states of the population, $\boldsymbol{n}_{r, 1} 984 \mathrm{r}=1, \ldots, 4$. This is dealt with in the next section.

\subsection{Prior distributions}

The state-space model contains 10 parameters: $\phi_{\mathrm{a}}$ (adult survival), $\phi_{\mathrm{pmax}}$ (maximum pup survival), $\beta_{1}, \ldots, \beta_{4}$ (related to region-specific carrying capacity), $\rho$ (shape of density dependent survival function), $\alpha$ (fecundity), $\omega$ (adults per female) and $\psi$ (pup production estimate precision). British grey seals are relatively well studied and several independent studies provide information about plausible values for many of these parameters. One method to utilize this information is to fit the model within a Bayesian inferential framework, using informative prior distributions on the parameters. The specification of these distributions was given extensive consideration by members of SMRU and SCOS; this is described in Supporting Information and a summary is given below. The resulting prior distributions are given in Table 1 and shown in Figure S3 (Supporting Information).

The prior on adult (i.e. age $1+$ ) survival $\phi_{a}$ was based on analysis of the age structure of historical samples of culled adult seals, and on mark-recapture estimates from intensively studied breeding colonies at North Rona and Isle of May; it uses a scaled beta distribution to constrain survival to be 
between 0.8 and 0.97 , with a mean of 0.9 and standard deviation (SD) of 0.04 . The prior on pup survival $\phi_{\text {pmax }}$ was largely based on mark-recapture studies and is considerably broader than that for $\phi_{a}:$ a beta distribution with mean 0.62 and SD 0.2. These same studies led to the conclusion that the density dependent shape parameter $\rho$ is probably more than 1 , and it was given a fairly broad prior using a gamma distribution with mean of 10 and SD 5. For fecundity $\alpha$, information was considered on pregnancy rates in culled adults as well as observed and inferred fecundity rates at the intensively studied breeding colonies; a scaled beta distribution was used to constrain fecundity to be between 0.6 and 1.0 , with a prior mean of 0.83 and SD 0.09 . The prior on number of adults per female, $\omega$, was derived from a comparison of age-specific survival estimates from samples of culled seals and markrecapture studies, including studies of Canadian grey seals (den Heyer \& Bowen, 2017); a tight prior based on a right-shifted gamma distribution with lower bound 1.6, mean 1.7 and SD 0.02 was used. The observation precision $\tau$ parameter for regional pup production estimates was based on consideration of the precision of colony-level estimates (which for aerial survey data is obtained as part of pup production estimation); a moderately broad gamma distribution with mean of 140 and SD 97 was specified, which corresponds to assuming a prior mean coefficient of variation (CV) of pup production of $10 \%$ and SD 5 (i.e. $90 \%$ of the prior probability density between $5 \%$ and $20 \%$ ).

To aid specification of priors on the $\beta$ parameters, a reparameterization was used. This is because the number of pups at carrying capacity ("carrying capacity of pups") is a function of both $\beta$ and $\rho$ (as well as other parameters, Figure S2). Carrying capacity of pups is strongly affected by $\rho$, so a prior distribution on $\beta$ that would produce a sensible prior on carrying capacity of pups at one level of $\rho$ would be infeasible at a different $\rho$. Hence, instead of setting priors on the $\beta$ for each region independently of the values of the other parameters, a reparameterization was used to set priors on the carrying capacity of pups per region, which then generated priors for the regional $\beta$ parameters, conditional on the priors for all the other model parameters. Denoting the carrying capacity of pups in region $r$ as $\chi_{r}$, it can be shown (by making use of the fact that the long-term age structure at carrying capacity is constant) that 
The prior distributions on the $\chi$ parameters were gamma distributions with means based loosely on the observed pup production trajectory but with large CVs of $50 \%$, and so were designed to be moderately uninformative.

As well as the model parameters, prior distributions are required on the hidden states, $\boldsymbol{n}_{r, t}$. The structure of the population dynamics model, where distribution of states at time $t$ is a function of that at time $t-1$, means that priors need only be specified on the states in the first year, $\boldsymbol{n}_{r, 1} 98 \dot{4}$ this (together with the priors on model parameters) then automatically specifies priors on all other states. To derive priors for $\boldsymbol{n}_{r, 1} 984$ the first year of pup production estimates $y_{0, r, 1} 984$ was used, meaning that during model fitting only observations from 1985 onwards were used. Pup production was assumed to be distributed according to

$$
n_{r, 0,1} 984 \mathrm{~N}\left(\mathrm{y}_{0, \mathrm{r}, 1984}, \frac{\mathrm{y}_{0, \mathrm{r}, 1984}^{2}}{\psi}\right)
$$

$$
n_{r, 0,1984} \mathrm{U}\left(\frac{\mathrm{n}_{\mathrm{r}, 0,1984}^{*}}{1.3}, 1.3 \mathrm{n}_{\mathrm{r}, 0,1984}^{*}\right)
$$

where $\mathrm{U}(a, b)$ denotes a uniform distribution with limits $a$ and $b$. Eqn. (4) is effectively "reversing" the observation equation, while Eqn. (5) adds further dispersion to the prior to help ensure it encompasses all likely values of pup production. The value 1.3 was chosen by trial and error: starting with a value of 1.0, multiple realizations were simulated from the prior parameters and the population was projected forwards through the time series; the multiplier was increased until the range of pup production values in the simulations for each region covered at least twice the range considered by the observers to be plausible for true pup production. (Larger values of the multiplier were also tried, and results found to be insensitive to this change, although the computational algorithm was substantially less efficient.) 
The prior distribution on age 1 females was derived by considering the pup survival process:

$$
n_{r, 1,1} 984 \operatorname{Bi}\left(\mathrm{n}_{\mathrm{r}, 0,1984}, 0.5 \phi_{\mathrm{p}, \mathrm{r}, 1984}\right)
$$

(the value of 0.5 is because only half of the pups are expected to be female). Similarly, the prior on age 2-5 females came from the adult survival process:

$$
n_{r, x, 1} 984^{\mathrm{Bi}}\left(n_{r, x-1,1984} 0.5 \phi_{a}\right) \quad x=2, \ldots 5 .
$$

Lastly, the prior on age $6+$ females came from "reversing" the fecundity process:

$$
n_{r, 6,1} 98 \sim \mathrm{Nb}\left(\mathrm{n}_{0, \mathrm{r}, 1984, \alpha}\right)+n_{0, r, 1984}
$$

The first term, $\mathrm{Nb}\left(\mathrm{n}_{0, \mathrm{r}, 1984}, \alpha\right)$, represents females that did not give birth, and the second, $n_{0, r, 1} 984$ represents females that did. $\mathrm{Nb}(r, p)$ denotes a negative binomial distribution, giving the number of failures before $r$ successes occur, where probability of success is $p$.

\subsection{Model fitting}

Fitting Bayesian state-space models of wildlife population dynamics, such as the one given above, typically relies on computer-intensive Monte Carlo simulation procedures (Chapter 4 in Newman et al. 2014). Two main approaches are Markov chain Monte Carlo (MCMC) and particle filtering (also called sequential importance sampling (SIS) or sequential Monte Carlo (SMC)). There are many variants of both approaches, indeed some that combine elements of both. Here, a modified version of the particle filtering method developed by Lui and West (2001) was used. Full details and computer code (in ANSI standard C for the particle filter, and R for the post-processing steps) are given in Supporting Information; a summary is given below. For a non-technical overview, see Thomas (2009).

\subsubsection{Particle filter}

A particle filter is an algorithm that produces a set of weighted random samples (particles) from a posterior distribution, in this case of a state-space model. Particles are generated from some initial 
distribution of states in the first time period, and are then projected forward stochastically through the time series. The weights relate to the manner in which the particles were sampled, how they were projected forward and the likelihood of the observed data given the simulated states. In the algorithm used here, the particles are initially generated by simulating from the prior distributions, and so each start with an equal weight (i.e. they are equally likely in the absence of any data). They are projected forward stochastically according to the population dynamics model (i.e. by simulating the survival, ageing and birth processes detailed in Section 2.3), and at each time-point the weight of each particle is updated by multiplying it by the likelihood of the observed pup productions in that year given the simulated pup production (the independent estimate of total population size is dealt with separately - see below). Hence, particles with simulated parameter values that produce population trajectories closely matching the observed pup productions will have high weight at the end of the simulation and vice versa. Indeed, it can be shown that the set of weighted particles form a weighted sample of the posterior distribution of the states and parameters. Inferences can readily be made from this weighted sample - for example to calculate the posterior mean population size one takes a weighted mean of the population sizes from the particles.

The above is the outline of a basic algorithm called importance sampling. In practice, however, this is inefficient since the prior distributions are diffuse relative to the posterior, so almost all the simulated population trajectories will be far from the observed pup production values and therefore have very low weight. Hence, additional procedures were added to the basic algorithm to improve efficiency, as follows.

Initial rejection control. The aim of this procedure is to weed out at an early stage sets of parameter and state combinations that are simulated from the prior but clearly have very low density in the posterior, so that computer time can be focused on areas of parameter and state space that have higher posterior density. Sets of 1,000,000 particles were simulated from the prior distributions, 
Rejection control was then applied - this is an algorithm that probabilistically removes particles with low weight and increases the weight of the particles not removed to ensure no bias is introduced (Liu, 2001). Here, the mean of the particles' weights was used as the rejection control criterion, resulting in approximately a tenth of the particles being retained. This process was repeated until there were at least $1,000,000$ particles surviving the initial rejection control stage.

Auxiliary particle filter with kernel smoothing of parameters. This procedure (based on an algorithm by Liu and West (2001)) is designed to increase efficiency by projecting forward deterministically at each time step and preferentially selecting those particles that produce states close to the observations, before then undertaking the stochastic projections (which are more computer intensive). Starting in 1985, particles were projected forward one time step, using expected outcomes from the population dynamics model rather than stochastic simulation. Observation weights were then calculated, and the particles were sampled with replacement ("resampled") using these weights - i.e. particles with high weights could appear multiple times in the resulting set of particles while those with low weights would tend to disappear. Instead of resampling with probability strictly proportional to the weights, the resampling probability used was proportional to the weights to the power of 0.25 . This "tempered resampling" means that the particles become less focused on the current and past data, and retain more diversity to better cope with future data points that may not match the current parameter estimates (Liu, 2001). This helps in the current dataset because later data points, where the growth trajectory is levelling out, are much more strongly indicative than the early data points that the carrying capacity of pups is lower than suggested by the priors (in 3 out of 4 regions at least).

Resampled copies of the same ancestor particle will have the same parameter values, so to maintain parameter diversity a second part to the Liu and West (2001) algorithm involves using kernel smoothing to jitter (i.e. add small amounts of random noise to) the parameter values. This can cause bias (Newman et al., 2006), so the amount of kernel smoothing was kept to a minimum, using a 
discount value of 0.99997 (a value of 1.0 results in no jittering at all). After kernel smoothing, particles were projected forward stochastically from 1985 to 1986 , and weights were adjusted to take account of the initial resampling. For reasons of numerical stability, weights were standardized so their mean was close to 1 . This procedure was repeated for all remaining years.

Final year rejection control. After the last year (2010), rejection control was used to reduce the number of particles to be stored. The rejection control criterion was 100 , which reduced the number of particles stored per run from 1,000,000 to around 50,000.

Multiple runs. The above procedures generated samples based on 1,000,000 particles (although fewer were stored after the final rejection control). However, even this many samples gave an imprecise estimate of the posterior distributions of interest. Hence, multiple runs were used to reduce Monte Carlo error (i.e. differences in results that would occur if the simulation procedure was performed again) to negligible levels. Note that standardization of the weights was done using the same standardization factors in all runs, so the weights remained comparable across runs.

In the results presented here, 4,000 runs were used, running in parallel in batches of 20 on two multiprocessor computers. To reduce the resulting outputs to a manageable level for post-processing (i.e. calculating posterior distributions on quantities of interest), it was necessary to apply further rejection control, this time using a rejection control criterion of 1,000 . This reduced the number of particles stored by a factor of approximately 10 .

\subsubsection{Post-processing}

The particle filter yields a set of weighted samples from the posterior given pup production data from 1984-2010. The first step in post-processing these samples was to incorporate the independent estimate of total population size, from 2008. This could have been done as part of the particle filtering but that would not have allowed a comparison of the estimates based on both pup production and the independent estimate with those based on pup production alone. To incorporate the independent 
estimate, the particles were re-weighted by multiplying their weights by the likelihood of the observed estimate (which was assumed to follow a gamma distribution) given the state value $N_{20}{ }_{0}{ }_{8}$ Summaries of the posterior distributions of the states (pups and adults) and parameters were then computed, both with and without the 2008 independent estimate of total population size.

Although the analysis used purposefully informative prior distributions on model parameters, it is of interest to know how much the posterior distribution of the parameters was influenced by the data and model, and how much by the priors. To this end, the percentage overlap between the marginal prior and posterior distribution for each parameter was calculated, as suggested by Garrett and Zeger (2000). High percentage overlap indicates parameters that are strongly influenced by the prior while low overlap indicates parameters strongly influenced by the data and model. Garret and Zeger (2000) suggested an ad hoc threshold of $35 \%$ in the context of non-informative priors. Although the current application is different from that envisaged by Garrett and Zeger, because the priors are designed to be informative, this threshold is still useful for evaluating which parameters are strongly influenced by the informative priors and which are not.

The population dynamics model is stochastic, in the sense that it includes demographic stochasticity by allowing survival and reproduction to be binomial random variables. Demographic stochasticity can have a large influence on population trajectory at small population sizes (tens of individuals) but given the size of the seal population, the estimated trajectory is likely to be dominated by the deterministic component of the model. Hence it is of interest to study the properties of the model from a deterministic perspective, which allows some simple mathematical analysis (Caswell, 2001). Specifically, at low population size the population grows exponentially, with the growth rate ("intrinsic rate of growth") given by the dominant eigenvalue of the Leslie matrix given in Eqn. (2) except with eigenvector associated with this eigenvalue. As the population increases, pup survival decreases until at carrying capacity (assuming the population reaches this limit), pup survival is given by 
416 (This expression is obtained by setting $\mathrm{n}_{0, \mathrm{r}, \mathrm{t}-1}$ to $\chi_{\mathrm{r}}$ in Eqn. (1), substituting $\beta_{r} \chi_{\mathrm{r}}$ into Eqn. (3) and 417 simplifying.) At carrying capacity, growth rate is zero, and the stable age structure is given by the eigenvector associated with the dominant eigenvalue of the Leslie matrix with $\phi_{p c c}$ in place of $\phi_{p, r, t}$. These quantities were calculated using the posterior mean parameter estimates as input.

Since the fitting method is based on Monte Carlo (MC) simulation, it is important to evaluate the level of $\mathrm{MC}$ error. For equally-weighted samples drawn independently from the posterior distribution, $\mathrm{MC}$ error is inversely proportional to the number of samples (note that this is samples from the posterior simulation - i.e. particles, not sample size of data). In the case where, as here, the samples are not equally weighted, the equivalent measure is the effective sample size (ESS):

$$
\mathrm{ESS}=\frac{k}{1+\mathrm{CV}(w)^{2}}
$$

where $k$ is the number of samples and CV(w) is the coefficient of variation of the weights (Liu, 2001). However, the samples are also not independent, because at the resampling stage of the particle filtering algorithm the same particle may be resampled multiple times. A highly conservative measure of ESS is to count multiple copies of the same particle as one single sample, so that $k$ is the number of unique ancestral particles. This measure was computed from the posterior sample. As an additional, more direct, check of $\mathrm{MC}$ error, the posterior summary statistics were computed separately for the first 2,000 runs of the particle filter and the second 2,000 runs and compared.

\section{Results}

\subsection{Input data}

The pup production data (circles in Figure 1) showed an increasing trend of approximately $7 \%$ per year in all regions up until the mid-1990s. This increase slowed and then stopped quite abruptly in three 
out of four regions - Outer Hebrides (slow-down starting early to mid 1990s), Inner Hebrides (mid 1990s) and Orkney (early-mid 2000s). Pup production in the fourth region (North Sea) continued to grow approximately exponentially. These patterns can be more readily seen on the log scale (Figure S4). Combining the four regions (Figure S5), the overall pattern is dominated by the two biggest pup production regions (Orkney and Outer Hebrides), showing an approximately exponential increase up until the mid-1990s and a decreasing rate of growth thereafter.

The scaled bootstrap estimates of total population size in 2008 had a mean 94,390 and SD 9,787. This was well approximated by the fitted right-shifted gamma distribution (Figure S6), which had a mean 94,398 and SD 9,788.

\subsection{Population size and trend}

Posterior mean estimates of pup production from the state-space model match the general trajectory in each region quite closely (Figures 1 and S4). Pup production is estimated to have peaked in the Outer Hebrides in 1998 before declining slightly and then levelling off at the end of the time series. In Inner Hebrides, pup production is estimated to have peaked in 2002 followed by a slight decline. In Orkney, the peak year is estimated to have been 2007, with the decline starting thereafter. In North Sea a near-exponential increase is estimated. The fits to pup production data from models with and without the 2008 independent estimate of total population size are nearly identical even in the years around 2008 (Figure 1), indicating that there is little information in the UK-wide estimate of total population size to inform region-level pup production.

There is some evidence of temporally correlated over- or under-prediction of pup production, reflecting the model's inability to reflect short-term trends in pup production. For example, in North Sea all eight estimates from 2001-2008 fall below the posterior mean (thick line in Figure 1); in Orkney the 13 estimates from 1993-2005 are all above the posterior mean. 
When the four regions are combined (Figure S5), it is clear that there is some slowing down in growth rate even from the beginning of the time series (see bottom panel of Figure S5, which is log scaled), but that the decrease in growth rate becomes more pronounced through time.

By contrast with pup production, the estimates of adult (i.e. age $1+$ ) population size are strongly influenced by the single independent estimate of total population size (Figure 2). The estimate for 2008 based on pup production data alone is substantially higher than the independent estimate posterior mean 124,500 (95\% credible interval (CI) 92,600-162,200), compared with independent estimate of 94,300 (results rounded to the nearest 100 seals). The estimates based on both pup production data and the independent estimate are a compromise between the two, and for 2008 is 102,200 (95\% Cl 87,000-121,600). Precision of the estimates is considerably improved by adding the 2008 independent estimate (compare credible interval widths in Figure 2).

The overall trajectory in adult population size is like that for pups, with a gradually decreasing rate of growth (Figure 2 and S7.) At the region level (Figures S8 and S9), the patterns also mimic those in pup production, with near-exponential growth in North Sea and a peak followed by slight decline in the other regions. However, the levelling off appears to occur 5 years earlier: 1993 in Outer Hebrides, 2002 in Inner Hebrides, and 2007 in Orkney.

One analysis goal is to estimate population size in the most recent time period, 2010. Population size of adults associated with regularly-monitored colonies was estimated to be 104,000 with $95 \%$ credible interval (Cl) 88,100-124,100 (Table S2). For that year, Duck \& Morris (2011) estimated that $89.53 \%$ of pup production took place in regularly-monitored colonies. Assuming the ratio of adults to pups at regularly-monitored and at other colonies is the same, the total British population size of adults (age $1+$ seals) in 2010 was 116,100 (95\% Cl 98,400-138,600). 


\subsection{Parameter estimates}

The marginal posterior parameter distributions (Figure 3 and Table 1) were, in general, strongly influenced by the priors. The prior-posterior overlap was greater than $35 \%$ for all parameters except three carrying capacity parameters (for Inner Hebrides, Outer Hebrides and Orkney). Nevertheless, posterior parameter distributions were somewhat altered from the priors in many cases.

The posterior mean on adult survival $\phi_{a}$ was higher than the prior mean (0.95 vs 0.90$)$, while that on maximum pup survival $\phi_{\text {pmax }}$ was lower (0.48 vs 0.62 ). These two parameters were moderately influenced by the prior distributions (prior-posterior overlap 35\% and $40 \%$ respectively). Looking beyond univariate marginal distributions to their bivariate relationship (Figure S10), $\phi_{a}$ and $\phi_{\mathrm{pmax}}$ were strongly negatively correlated in the posterior (Pearson's correlation coefficient $r=-0.85$ ).

Fecundity $\alpha$ was estimated to be higher in the posterior than the prior (means 0.90 and 0.83 respectively), although there was considerable (70\%) overlap between the marginal prior and posterior distributions. All of the information about fecundity came from the independent estimate, since the posterior distribution with pup production data alone was nearly identical to the prior (Figure S11).

The density dependence shape parameter, $\rho$, had a lower posterior than prior mean (5.95 vs 10 respectively) - this still implies a moderately steep drop in pup survival with increasing population size (Figure S2). For the pup production at capacity parameters, $\chi$, the posterior distribution for North Sea was somewhat similar to the prior (posterior mean 15,500; overlap with prior 77\%) and much higher than the current estimated pup production (around 8,000); for the other regions there was strong evidence that all three have reached carrying capacity, because the posterior distributions were centred on values close to the current estimates of pup production, with low SDs (Table 1) and low overlap with the priors (9\%-14\%). 
The observation precision parameter $\psi$ was only moderately altered from the prior (49\% overlap) with a similar mean but a smaller SD.

Lastly, the sex ratio parameter $\omega$ was unchanged by the fitting process ( $99 \%$ prior-posterior overlap), suggesting that there is no information in the data or model above that provided in the prior distribution.

\subsection{Eigenanalysis}

Using the posterior mean parameter estimates, the intrinsic rate of population growth was estimated as $7.1 \%$ per year, with a stable age structure comprising $33 \%$ pups, $30 \%$ age $1-5$ adult (nonbreeding) females and $37 \%$ age $6+$ (i.e. breeding-age) females (Table S3) (adult males do not feature in the Leslie matrix). At carrying capacity, pup survival declined from the maximum $\phi_{\mathrm{pmax}}=0.48$ to $\phi_{p c c}=0.14$. The stable age structure at carrying capacity was $41 \%$ pups, $12 \%$ age $1-5$ adult females and $46 \%$ age $6+$ females (i.e. breeding age). Note that the ratio of pups to breeding females is unchanged (as one would expect in a model with constant fecundity), but the proportion of pre-reproductive females has declined substantially.

\subsection{Monte Carlo error}

In total, $4 \times 10^{8}$ particles were simulated after the initial rejection control stage, of which $2 \times 10^{7}$ were retained after the final rejection control. At that stage, the effective sample size (ESS) of unique ancestral particles was 1669; after reweighting according to the independent estimate of total population size the final ESS was 478.

Dividing the sample of particles in half and recomputing the results separately for each half indicated that all estimates of posterior mean and SD for states and parameters are accurate to at least 3 significant figures. 


\subsection{Population trends}

529 Overall annual population growth is estimated to have slowed considerably in recent years, going from approximately $6 \%$ in $1984-5$ to $<1 \%$ since 2002 (Figure 2 , Table S2). The trajectory varies considerably among the four regions (Figure S8). Outer Hebrides, Inner Hebrides and Orkney are estimated to each in turn have slowed their growth quite suddenly, slightly overshot their carrying capacity and undergone a small decline thereafter. By contrast, the North Sea region continued to grow at a nearconstant rate and by 2010 is estimated to have a pup production that is approximately half way to carrying capacity (estimated pup production in $2010=8,119$; posterior mean $\chi_{4}=15,500$ ). However, the estimate of pup production at carrying capacity in North Sea is understandably imprecise $\left(\chi_{4} 95 \%\right.$ $\mathrm{Cl} 6,976-35,620$ ) and with posterior distribution quite close to the (fairly diffuse) prior. This is expected since it is not feasible to determine where the asymptote is of a trajectory that is still strongly in the growth phase. All three of the other regions saw pup production grow strongly before quickly levelling off, further emphasizing that one cannot tell whether the North Sea region is close to carrying capacity or far from it.

In each of the three regions at carrying capacity, adult population size is estimated to have reached its peak 5 years before pup production peaked (cf. Figures 1 and S5). This is because the adult population as defined here is made up of aged 1-5 non-breeding females, as well as aged 6+ breeding-age females: as population size increases there comes a point at which the survival of pups declines dramatically (see Figure $\mathrm{S} 2$, bottom panel, for values of $\rho$ in the range 5-7), causing a decline in aged 1 females and reduction in pup production. 
Such delays between density dependence affecting young animals and the effect becoming evident in the breeding population are well known to produce damped oscillations and even stable population cycles under some circumstances (e.g. Turchin, 2003). Here, the extended Beverton-Holt formulation allows pup production to decline relatively sharply once the decline starts (posterior mean estimate leading to the estimated overshoot in both adult population size and pup production - i.e. a damped oscillation.

It is important to remember that there is no time series of observations of adult (age $1+$ ) population size, and hence the inferences about density dependence and damped oscillations are heavily reliant on the model structure. Although the model appears to fit well to both pup production data and the single independent estimate of total population size, the inference about population processes is still indirect.

\subsection{Historical context and management applications}

563

The results show that the UK grey seal population has increased continuously throughout the 25-year monitoring programme presented here. This is a continuation of a long-term pattern of increase since the first systematic attempts to estimate the UK populations and formal surveys began in the 1960s (Summers 1978). Indeed, historical references to very small local populations and the widespread occupation of many island breeding colonies by farming communities, means that the current population probably represents the highest population since colonisation of the Northern and Western Isles during the Neolithic. There have been no large-scale mortality events of the type seen during the PDV epidemics in UK harbour seals and, again unlike harbour seals, there have been no regional declines.

Growth rates have varied across the four regions, with the western and northern Isles breeding populations all apparently at or approaching carrying capacities. The population in the North Sea has 
continued to grow almost exponentially. Russell et al. (submitted) show that in the later years of the time series this increase is associated with particularly rapid increases in pup production at sites in the southern North Sea and mainly at sites on the mainland coast in areas that would not have been available to grey seals until relatively recently.

Timely estimates of British grey seal population size and trend are required by NERC to discharge its statutory requirement to advise on seal management. A standard question to SCOS each year is "What are the latest estimates of the number of seals in UK waters?" (Special Committee on Seals 2017, p.10). The outputs of the population dynamics model has other management uses, described briefly here.

Estimated population size is essential for converting diet composition data to total prey consumption - something of strong interest in fisheries management. In the UK population estimates have been used to estimate consumption to assess the importance of grey seal predation as a mortality factor in large gadoid stocks (e.g. Cook, \& Trijoulet, 2016; Hammond \& Wilson, 2016; Smout, Rindorf, Hammond, Harwood, \& Matthiopoulos, 2014; Trijoulet, Holmes, \& Cook, 2017).

Assessing the consequences of removals from a population requires accurate estimates of total population size as well as estimates of demographic parameters; e.g. grey seals in Scotland are managed on the basis of the Potential Biological Removals (PBR) method that uses up-to-date estimates of the population size and an estimate of the confidence intervals around the estimate to calculate appropriate maximum safe removal levels. The population model described here is also ideal for application of more complex management approaches based on Population Viability Analysis (PVA). In general, the population model represents the only currently available, non-lethal method for estimating population wide survival and fecundity rates.

The population models presented here do not differentiate between sub-populations at the colony level. Grey seals are the primary reason for designation for six Special Areas of Conservation (SACs) 
encompassed by the four modelled populations. These SACs consist of either single or small groups of colonies so at present, pup production estimates are used as the indices for assessing conservation status at the small scales (Russell et al., submitted). However, pup production is more an indicator of population productivity and could vary significantly with changes in fecundity and/or local redistribution. Trends in the overall population size are therefore a more robust and stable indicator of population status and are required to provide a context for these shorter-term production indices.

\subsection{Model adequacy and extensions}

605

606

607

608

609

As noted earlier, the overall estimated trajectory of pup production in each region is a good fit to the trends in the data. However, there is evidence of some temporally correlated over- or underprediction of pup production (Figure 1). There is also some evidence of higher dispersion about the fitted trajectory at higher pup production levels, especially in Outer Hebrides. (Note, however, that this is expected to some extent if the observation model, which specifies a constant CV on observation error, is correct.) One possible model extension to account for these issues would be to add a random effect on fecundity, potentially allowing fecundity to follow a random walk reflecting correlated changes in environmental conditions over time. This could be specified at the global level, or separately by region, possibly with a shared fecundity variance parameter. (There is evidence for variation in fecundity between two intensively-sampled colonies in different regions, Smout, King \& Pomeroy, submitted; see also below.) An alternative would be to specify a random effect on carrying capacity, although the variation in pup survival that this would produce would not lead to strong annual fluctuations in pup production since recruiting females make up only a small proportion of the breeding-age population (Table S3: age 5 females are around an order of magnitude fewer than age $6+$ females)

The estimated total population size in 2008 , based on the population model and pup production data, was $32 \%$ higher than the independent estimate (Figure 2). Two model parameters most directly translate pup production into total population size: fecundity $\alpha$ and number of adults per female $\omega$. 
623 Fecundity is important because the number of breeding-age (i.e. $6+$ ) females is equal to pup production divided by fecundity, and breeding-age females make up over half the adult (i.e. age 1+) female population even when the population is growing exponentially (Table S3). Adults per adult female is important as it is a direct multiplier, converting the estimate of adult females from the statespace model into an estimate of total population size. The posterior distribution of both parameters ( $\alpha$ and $\omega$ ) is strongly influenced by the prior (completely so for $\omega$ ) emphasizing the importance of carefully specifying these distributions. This does not imply there is no information in the data about either parameter; rather that given the informative priors used, there is little additional information above that specified by the prior. Given the model structure, priors and data, it is not possible to say which parameter prior(s) might have been mis-specified to cause the $32 \%$ mis-match - although this could also be due to sampling error or an unquantified bias in the independent estimate.

The final population estimate is effectively a compromise between the estimate resulting from the population model informed by model priors and pup production data, versus the independent estimate of total population size from 2008. It can be thought of as a weighted average of the two, with the weighting depending on their relative precision. The estimate from the population dynamics model is relatively imprecise compared with the independent estimate (compare $\mathrm{Cl}$ widths in Figure 2), and so the final estimate is closer to the 2008 independent estimate than the estimate from the population model - although of course the population model provides the trajectory. Changes in model priors could affect this balance: in particular the assumptions about precision of the pup production data can be expected strongly to affect precision of the resulting population estimates. The observation precision parameter $\psi$ is currently estimated as part of model fitting. This is not ideal, since the observation error can be difficult to estimate correctly in state-space models, and misspecification can affect identification of density dependence (Knape, 2008). Pup production estimates are currently generated with estimates of uncertainty at the colony level (Russell et al., Submitted), but since colony estimates share parameters they are not statistically independent, making regionlevel estimates of uncertainty difficult to compute. This is a topic of future research. 
The posterior parameter estimates largely seem biologically plausible, as one would expect given the use of informative prior distributions on most parameters. The prior on adult survival has an upper cut-off at 0.97 , and the posterior has a mode close to this value (Figure 3). Recent estimates from a long-term brand re-sighting study on Canadian grey seals (den Heyer \& Bowen 2017) are higher than this cut-off ( 0.989 (SE 0.001) for females age $5-26$ by the definition of age used in this paper, 0.904 (0.004) for ages $26+$ and $0.976(0.001)$ overall). It may be necessary, therefore, to alter the prior in future runs of this model. Any resulting increase in the posterior distribution of adult survival is likely to be counterbalanced by a decrease in estimated maximum pup survival, since the two are strongly negatively correlated (Figure S10), with little resulting consequence for estimated population size. This is exactly the effect shown in a previous analysis (Thomas, 2013), which found that an unconstrained upper bound on the adult survival prior produced little change in population size but unrealistic posterior mean values: high adult survival (0.99), and low maximum pup survival (0.28). The negative correlation between these two parameters is a consequence of the fact that only one age class of seal (pups) is observed in each year; given the assumed population model, the observed pup production trajectory can be explained by high adult and low pup survival, or lower adult and higher pup survival. In the population dynamics model, adult survival, maximum pup survival and fecundity are all assumed to be the same across regions and constant over time. These assumptions may not be appropriate, but there is little information available at relevant spatial scales to suggest how they may vary over space and time. Regarding variation by region, Thomas (2013) fitted the model described here independently to each region (using just pup production data). The posterior distributions on all three parameters did not vary greatly between regions, although estimates of maximum pup survival and fecundity were largely driven by the prior. The estimate of adult population size in North Sea was somewhat higher and in Orkney was lower than from a global analysis; overall, the total population size was very similar to the global analysis. 
One source of information at the colony level comes from two breeding colonies, Isle of May (North

674 Sea) and North Rona (Outer Hebrides), that have been intensively studied over many decades. These studies were used to inform the prior distributions on the demographic parameters (see Supporting Information). As well as being in different regions, these colonies show markedly differing trends in pup production: pup production at the Isle of May increased at c. $10 \%$ p.a. before levelling off in the early 2000s, and at North Rona, pup production peaked in the early 1990 s and has since been declining at c. 5\% p.a. (Russell et al., Submitted). Thomas (2013) fitted separate models to these two regions using priors for fecundity in each region based on estimated fecundity for the corresponding intensively-studied colony within that region. Posterior distributions on fecundity were very similar to the priors and, since both had a lower mean than the prior used for the global analysis, the estimate of regional adult population size was higher (by 10-15\%). The prior on adult survival was extended below (0.8) the lowest estimates from these colonies (0.871; Smout, King \& Pomeroy, Submitted) because the parameter represents survival for the 1+ age class; the mean survival rate of this class may be lower than that of breeding females estimated for these colonies (see below). Smout, King \& Pomeroy (Submitted) found significantly lower adult survival at North Rona than at the Isle of May (values given in Table S4). Such estimates could be used conduct a similar exercise as that described above using fecundity estimates. However, it is unclear to what degree the lower survival at North Rona reflects lower survival for the Outer Hebrides (compared to the North Sea) or is the result of the higher proportion of older females (compared to the Isle of May) in the declining North Rona colony. In any case, the sensitivity analyses performed to date suggest that allowing regional variation in demographic parameters makes only a small difference to inferences about population size. trends in these parameters without more independent information about how they might vary, particularly for $\alpha$ and $\phi_{p \max }$ which are already strongly informed by their prior distribution. Evidence is emerging of variation in fecundity being driven by environmental variation (Smout, King \& Pomeroy, 
realistic to extend the model to allow for a random effect on $\alpha$, although without independent information about the observation error in pup production random variation in fecundity and observation error will be strongly confounded and so difficult to estimate together. It seems unlikely that random variation in either parameter will make a substantial difference to the estimate of population size.

704

The assumption of constant adult survival across all age groups is also questionable. Juvenile (age 1-

5) females may exhibit lower survival rates than breeding-age (6+) females. Starvation is a major cause of mortality for pups once they leave the beach, but not for adults (Baker, Jepson, Simpson, Kuikeen. 1998). It seems likely much of this occurs within the first few months at-sea; weaned pups have around a month to develop an effective foraging strategy before terminal starvation occurs (Bennett, Speakman, Moss, Pomeroy, Fedak, 2007. Pups appear to exhibit adult-like foraging trips within the first four-months at sea (Carter et al. 2017). Thus, although such individuals may face a degree of competitive exclusion from the most profitable foraging patches (Breed, Bowen, \& Leonard, 2013), there is no evidence that starvation-induced mortality would be higher in juveniles (1-5) compared to adults. However, there is evidence that juvenile seals may be more susceptaible to disease than adults as energy is directed to growth at expense of their immune system (Nymo et al., 2013). Conversely, it is also possible that initial attempts at breeding may lower survival (Smout et al., Submitted). Senescence (i.e. reduced survival rates of very old females) is also thought to occur, and an extensive analysis of Canadian data indicated lower survival rates for females aged $25+$ compared with those aged 4-25 (den Heyer \& Bowen 2017). Thomas (2015) investigated the effect of introducing senescence into the population dynamics model, by fitting preliminary data from the Canadian study to a Gomperz-Makeham hazard function. Almost no difference was found in the resulting population trajectories. This was attributed to the fact that senescent individuals make up a very small proportion of the adult breeding population. Currently, there is no basis for which to make juvenile survival different from adult, and in any case such a change would have little impact on population estimates, because of the negative correlation between pup and adult survival. 
Age at first reproduction was assumed fixed in the model at 6 , with fecundity assumed constant after that age. In practice, age at first reproduction appears to be variable with a small proportion of females breed at younger ages (e.g. Harwood \& Prime, 1978), and others at older ages (Pomeroy, Smout, Moss, Twiss, \& King, 2010). This is readily incorporated into the population dynamics model, as demonstrated by Thomas, Hammill, \& Bowen (2011) who modelled population dynamics of Canadian grey seals and included an age-specific fecundity parameter based on analysis of pregnancy rates in a sample of shot seals. There is some evidence that that age at recruitment may increase in a density dependent manner; Bowen, Iverson, McMillan, \& Bonness, 2006 have found that age at recruitment has increased by approximately one year over a 15 -year period. It is unlikely that including such detail, rather than the current single average fecundity parameter, will have a significant effect on the estimate of population size.

The prior distribution on adult survival and fecundity implies a prior mean on pup survival at carrying capacity ( $\phi_{p c c}$ in Eqn. 6 ) of 0.42 . The posterior distribution on adult survival and fecundity are both higher than the priors (Figure 3), resulting in a lower posterior mean $\phi_{p c c}$ of 0.14 ( $95 \% \mathrm{Cl} 0.08-0.26$ ). This level of pup mortality is high, but may be plausible given evidence for high levels of starvation in pups post-weaning (Baker, Jepson, Simpson, \& Kuikeen, 1998).

The population dynamics model specified here assumes that density dependence operates by reducing pup survival. Alternatively or in addition, density dependence could arise through an increase in age at first reproduction (see above), a decline in fecundity rate of breeding-age females or a decline in adult survival. As with all long-lived species, population growth rate is most sensitive to changes in adult survival, in the sense that a given absolute decrease in adult survival produces a greater reduction in growth rate than the same decrease in fecundity or pup survival (Turchin, 2003). Harwood and Prime (1978) demonstrated this for the grey seal population. Nevertheless, both theoretical and practical considerations mean that adult survival is likely to be the last demographic parameter to be affected by density-related intra-specific competition (see, for example, Bonenfant 
et al., 2009). Harwood and Prime (1978) considered the various mechanisms by which density dependence could operate on demographic parameters and concluded that the most likely was a decline in pup survival due to over-crowding on the breeding colonies. However, Twiss, Duck and Pomeroy (2003) found that pre-weaning pup mortality was not related to measures of density within the colony at North Rona. Russell et al. (Submitted) consider evidence from more recent studies and concur that pup survival is likely to be the primary target of density dependence; they provide suggestions as to further mechanisms operating in the first year of life and suggest that density dependence is likely operating at sea.

Density dependence operating through fecundity has been considered as an alternative model for the British grey seal population (Thomas, 2010; Thomas \& Harwood, 2008). Estimated adult population size from the state-space model is approximately twice as large under this model than a density dependent pup survival model, because density dependence causes fecundity to decline to a low level at carrying capacity, and the population of age $6+$ adults is given by pup production divided by fecundity. Instead of the population at carrying capacity being composed of a large number of pups that fail to survive their first year, it is composed of a large number of $6+$ females who fail to reproduce. The trajectory of pup numbers at the region level is similar from density dependent survival and density dependent fecundity models (Thomas, 2010), but nonetheless the survival model was favoured when posterior model probabilities were computed, based on pup production data alone. When the independent estimate of total population size was introduced, the density dependent survival model was favoured even more strongly because the density dependent fecundity model produced total population estimates that were far higher than the independent estimate. Further support comes from Smout et al. (Submitted), who found similar estimates of fecundity at the two intensively-studied colonies with contrasting pup production trajectories; declining at North Rona and increasing at the Isle of May (Table S5). 
The sex ratio parameter (number of adults per female, $\omega$ ) is assumed constant between regions and over time. One way this could change is if density dependence operates differentially on different components of the population so that the survival of male pups changes with increasing population size at a different rate from the survival of female pups. Carter et al. (2017) found sex-specific differences in foraging strategies which differed by region. The drive to become a competitive adult male, rather than just survive, may result in an increasingly risky foraging strategy as population size increases and resources become limiting. This would cause $\omega$ to vary depending on population size.

An alternative to including adult males via the sex ratio parameter is to explicitly model their numbers as hidden states in the state-space model, in the same way that females are modelled. The sex ratio parameter would then be replaced by a parameter for adult male survival. This would allow external information about male survival rate or age structure to be incorporated more directly but has the disadvantage that the fitting algorithm would need to track six more states per region and year (assuming age 1-6+ males are tracked), increasing the computational burden.

The current model assumes all females breed in the region where they were born. The available evidence suggests that, for the most part, once recruited into a breeding colony, females remain faithful to it (Pomeroy, Twiss, \& Redman, 2000). Grey seals also exhibit a degree of natal philopatry, recruiting into the breeding population in which they were born. However, at least within a region, substantial dispersal into other breeding colonies would be required to explain the local trends in pup production (Gaggiotti et al., 2002; Russell et al., Submitted). Indeed, the North Sea consists of two geographically distinct groups of colonies, and in recent years the increase in pup production in the southern group could only be explained by recruitment of females born further north. Although there is evidence for recruitment of grey seals, born in the study area, into continental Europe (Brasseur et al. 2015), the loss to the UK grey seal pup population would be negligible. There is no direct evidence of movement of females between the regions considered here but it is possible that southwards 
movement of recruiting females may have contributed to the sharp slow-down in pup production in Orkney and the continued increase in the North Sea region.

A model allowing movement of recruiting females between regions has already been developed for the British seal population and fitted to pup production data (Thomas \& Harwood, 2003; Thomas et al., 2005). The model was based on a hypothesis of density dependent dispersal (Ruxton \& Rohani, 1998) and evidence for this in grey seals at the colony level (Gaggiotti et al., 2002). The model allowed recruiting females to move away from their natal region (after which they were again fixed), with movement probabilities proportional to the distance between target and natal regions and the difference in expected pup survival between target and natal regions. Tendency to remain in the natal region was represented in the model as a site fidelity parameter. Later research (e.g. Thomas \& Harwood, 2009) found that this model had lower posterior model probability than models without movement, but it would be worth re-visiting the movement models in the light of more recent data, and also re-considering the prior distributions placed on movement model parameters. Preliminary work using a Europe-wide dataset did support a movement model at that level (Russell, Hanson, \& Thomas, 2016).

\subsection{Fitting algorithm}

The particle filtering algorithm implemented here is generally applicable to other models of wildlife population dynamics. All that is required to implement a new model is to be able to simulate from

817 the prior distributions of model parameters and initial states, to project the simulations forward stochastically in time and to evaluate the likelihood of observations given the population values. This ready ability to adapt the algorithm to diverse models has been termed "plug and play" (He, lonides, \& King, 2010). designed to maintain the "plug and play" nature of the algorithm, while coping with the fact that the 
state-space model contains a number of time-invariant parameters $\left(\phi_{a}, \phi_{p \max }\right.$, etc.), which makes many particle filtering algorithms fail. A disadvantage of the Liu and West (2010) algorithm is that it produces biased estimates of the posterior distribution (Newman et al., 2006). This bias can be minimized by appropriate selection of a tuning parameter (see Section 2.6.1), at the expense of additional computational effort. For the runs in this paper, the tuning parameter was set conservatively and so bias is likely to be negligible, although computational effort was high.

One downside, therefore, of the algorithm is that it is computer-intensive: the runs reported here took approximately 3 days to complete, running as 20 parallel processes. One alternative is to use a Markov chain Monte Carlo (MCMC) algorithm, for which software exists that allows the analyst to specify the statistical model and leave details of the algorithm implementation up to the software. Giminez et al. (2008) provide an introduction, focusing on the BUGS software, that includes a state-space population dynamics model example; some more recently-developed software is listed by Goudie et al. (2017).

Although off-the-shelf MCMC software will work well for some state-space population models, currently-available software performs poorly for the seal model because of the model structure. The fact that only the most junior age class is observed induces strong correlations among all other age classes and across time, making efficient sampling of the posterior state distributions difficult with standard MCMC approaches. Newman et al. (2006) developed a custom MCMC algorithm, which was relatively efficient but was highly tuned to the seal model. They compared it to the particle filtering algorithm applied to the same problem and found that while the particle filter was somewhat slower, the ease with which new models could be implemented meant it would be preferred in situations where the ability to readily explore new models was desirable.

844 As noted earlier, the development of efficient and general algorithms applicable to this type of model is an active area of research (e.g. Doucet \& Lee, in press; Kantas, Singh, Maciejowski, \& Chopin, 2015). In addition, computer hardware continues to improve in speed, and parallel processing architectures, for which particle filtering algorithms are particularly well suited, become increasingly accessible (e.g. 
Mingas et al., 2017). Extensions to the population model that are currently computationally difficult (such as implementation of a random effect on carrying capacity) will soon be entirely feasible.

An alternative model fitting approach was developed by Lonergan et al. (2011). Demographic parameters were estimated by finding key summary statistics that could be obtained from the pup production data (e.g. maximum rate of increase) and using approximate Bayesian computation to match the summary statistics to the data. Although the method involves approximating the full population model, it has the strong advantage of being much less computationally intensive, and is therefore useful for exploratory analysis and for larger datasets.

\subsection{Conclusion}

By constructing a plausible population model for British grey seals based on data available at the time, Harwood and Prime (1978) concluded that, while the population was growing exponentially at about $7 \%$ per year one could estimate total population size by multiplying the measured pup production by 4 (their Figure 6; note that total population size in that paper included pups). They further argued that density dependence would operate by reducing pup survival, and that in this case, the multiplier would go down to 3 as the population growth rate approached zero. Forty years later, we have the benefit of much more information about the demographic parameters, an observed levelling-off of pup production, an independent estimate of population size and more sophisticated modelling techniques. The analysis presented here suggests that the appropriate multiplier for the period of near-exponential growth in the 1980s was approximately 4.5 (e.g. for 1984: posterior mean adult population size associated with regularly monitored colonies was 53,000 , and pup production was $14,992$, so the multiplier is $(53,000+14,992) / 14,992)$. For the three regions that are now close to carrying capacity (although a stable age structure has likely not yet been reached), the multiplier is approximately 3.4 (e.g. for 2010: Inner Hebrides $(7,400+3,108) / 3,108=3.4$; Outer Hebrides 


\section{Acknowledgements}

874 The authors thank all those responsible for collection and processing the long-term data used in this

875 paper. Thanks to Ken Newman and Steve Buckland for their contributions to the modelling framework and fitting methods, Jason Matthiopoulos, Paddy Pomeroy, Ailsa Hall, lan Boyd and members of the Special Committee on Seals for many helpful suggestions and insightful discussions regarding the seal population model and the prior distributions. We are particularly grateful to the editor, John Baxter, and two referees, Jeremy Greenwood and Don Bowen, for their detailed comments, which led to a greatly improved paper. The work reported here was part-funded by the UK Natural Environment

Baker, J. R., Jepson, P. D., Simpson, V. R., \& Kuikeen, T. (1998). Causes of mortality and non-fatal conditions among grey seals (Halichoerus grypus) found dead on the coasts of England, Wales and Isle of Man. Veterinary Record, 142, 595-601.

Bennett KA, Speakman JR, Moss SEW, Pomeroy PP, Fedak MA. 2007 Effects of mass and body composition on fasting fuel utilisation in grey seal pups (Halichoerus grypus Fabricius): an experimental study using supplementary feeding. J. Exp. Biol. 210, 3043-3053.

Beverton, R.J.H. \& Holt, S.J. (1957). On the Dynamics of Exploited Fish Populations. London, UK: H.M 890 Stationary Office.

Borchers, D.L., Buckland, S.T., \& Zucchini, W. (2002). Estimating Animal Abundance. London, UK: Springer. (2009). Advances in Ecological Research 41. London, UK: Elsevier. 
Bowen, W.D., Iverson, S.J., McMillan, J.I. \& Boness, D.J. (2006) Reproductive performance in grey seals: age-related improvement and senescence in a capital breeder. Journal of Animal Ecology, 75, $1340-1351$.

Brasseur, S. M. J. M., van Polanen Petel, T. D., Gerrodette, T., Meesters, E. H. W. G., Reijnders, P. J. H., \& Aarts, G. (2015). Rapid recovery of Dutch gray seal colonies fueled by immigration. Marine Mammal Science, 31, 405-426.

Breed, G. A., Bowen, W. D., \& Leonard, M. L. (2013). Behavioral signature of intraspecific competition and density dependence in colony-breeding marine predators. Ecology and Evolution, 3(11), $3838-3854$.

Carter, M. I. D., Russell, D. J. F., Embling, C. B., Blight, C. J., Thompson, D., Hosegood, P. J., \& Bennett, K. A. (2017). Intrinsic and extrinsic factors drive ontogeny of early-life at-sea behaviour in a marine top predator. Scientific Reports, 7(1), 15505.

Caswell, H. (2001). Matrix Population Models, $2^{\text {nd }}$ Edition. Sunderland, USA: Sinaur Associates.

Cook, R. M., \& Trijoulet, V. (2016). The effects of grey seal predation and commercial fishing on the recovery of a depleted cod stock. Canadian Journal of Fisheries and Aquatic Sciences, 73(9), 1319-1329.

den Heyer, C., \& Bowen, W. D. (2017). Estimating changes in vital rates of Sable Island grey seals using mark-recapture analysis. DFO Canadian Science Advisory Secretariat Research Document 737 2017/054, 27. Canada: Department of Fisheries and Oceans.

Doucet, A. \& Lee, A. (In press). Sequential Monte Carlo methods. Chapter 7 in Maathuis, M., Drton, M., Lauritzen, S., \& Wainwright, M. (Eds.) Handbook of Graphical Models. London, UK: Chapman \& Hall/CRC Press. 
Duck, C.D. (2009). Grey seal pup production in Britain in 2008. SCOS Briefing Paper 09/01. http://www.smru.st-andrews.ac.uk/research-policy/scos/ [20 Aug 2018]

Duck, C.D. \& Morris, C.D. (2011). Grey seal pup production in Britain in 2010. SCOS Briefing paper 11/01. http://www.smru.st-andrews.ac.uk/research-policy/scos/ [20 Aug 2018]

Gaggiotti, O.E., Jones, F., Lee, W.M., Amos, W., Harwood, J., \& Nicols, R.A. (2002). Patterns of colonization in a metapopulation of grey seals. Nature, 416, 424.

Garrett, E.S. \& Zeger, S.L. (2000). Latent class model diagnosis. Biometrics 56: 1055-1067.

Giminez, O., Bonner, S., King, R., Parker, R.A., Brooks, S., Jamieson, L.E., ... , \& L. Thomas. (2008). WinBUGS for population ecologists: Bayesian modeling using Markov Chain Monte Carlo methods. Pp 885-918 in Thomson, D.L., E.G. Cooch and M.J. Conroy (Eds.) Modeling Demographic Processes in Marked Populations. Environmental and Ecological Statistics Vol 3. London, UK: Springer.

Goudie, R.J.B., Turner, R.M., De Angelis, D., \& Thomas, A., (2017). MultiBUGS: Massively parallel MCMC for Bayesian hierarchical models. eprint arXiv:1704.03216.

Hall, A. (Submitted) Preface to this special issue.

Hammond, P., \& Wilson, L. (2016). Grey seal diet composition and prey consumption. Scottish Marine and Freshwater Science, 7, 20-47.

Harrison, P.J., Buckland, S.T., Thomas, L., Harris, R., Pomeroy, P.P., \& Harwood, J. (2006). Incorporating movement into models of grey seal population dynamics. Journal of Animal Ecology, 75, 634645.

Harwood, J. \& Prime, J.H. (1978). Some factors affecting the size of British grey seal populations. Journal of Applied Ecology 15: 401-411. 
Harwood, J., and Greenwood, J.J.D. (1985). Competition between British grey seals and fisheries. Pp 153-169 in Beddington, J.R., Beverton, R.J.H. and Lavigne, D.M. (Eds.) Marine mammals and fisheries. George Allen and Unwin, UK: London.

He, D., lonides, E.L., \& King, A.A. (2010). Plug-and-play inference for disease dynamics: Measles in large and small towns as a case study. Journal of the Royal Society Interface, 7, 271-283.

Kantas, A.D., Singh, S.S. , Maciejowski, J.M. \& Chopin, N. (2015) On particle methods for parameter estimation in state-space models. Statistical Science 30: 328-251.

Knape, J. (2008) Estimability of density dependence in models of time series data. Ecology 89: 29943000. doi:10.1890/08-0071.1

Lambert, R.A. (2002) The grey seal in Britain: A twentieth century history of a nature conservation success. Environment and History 8: 449-474.

Lonergan, M.E., Duck, C.D., Thompson, D., Moss, S. \& McConnell, B.J. (2011) British grey seal (Halichoerus grypus) abundance in 2008: an assessment based on aerial counts and satellite telemetry. ICES Journal of Marine Science, 68, 2201-2209.

Lonergan, M., Thompson, D., Thomas L. \& Duck, C. (2011). An approximate Bayesian method applied to estimating the trajectories of four British grey seal (Halichoerus grypus) populations from pup counts. Journal of Marine Biology, Article ID 597424.

Liu, J.S. (2001). Monte Carlo Strategies in Scientific Computing. New York, USA: Springer-Verlag.

Liu, J. \& West, M. (2001). Combining parameter and state estimation in simulation-based filtering. Pp 197-223 in A. Doucet, A., de Freitas, N. \& Gordon, N., Sequential Monte Carlo Methods in Practice. New York, USA: Springer-Verlag. 
Mingas, G., Bottolo, L., \& Bouganis, C-S. (2017). Particle MCMC algorithms and architectures for accelerating inference in state-space models. International Journal of Approximate Reasoning, 83, 413-433.

Newman, K.B., Buckland, S.T. , Lindley, S.T. , Thomas, L., \& Fernández, C. (2006). Hidden process models for animal population dynamics. Ecological Applications, 16, 74-86.

Newman, K., Buckland, S.T., King, R., Borchers, D.L., Cole, D., Besbeas, P., Gimenez, O., \& Thomas, L. (2014). Modelling Population Dynamics: Model Formulation, Fitting and Assessment using State-Space Methods. London, UK: Springer.

Nymo, IH, Tryland, M, Frie, AK, Haug, T, Foster, G, Rodven, R, Godfroid, J (2013). Age-dependent prevalence of anti-Brucella antibodies in hooded seals Cystophora cristata. Diseases of Aquatic Organisms 106 (3):187-U16

Pomeroy, P., Smout, S., Moss, S., Twiss, S. \& King, R. (2010). Low and Delayed Recruitment at Two Grey Seal Breeding Colonies in the UK. Journal of Northwest Atlantic Fishery Science, 42, 125133.

SCOS (2017) Scientific Advice on Matters related to the Management of Seal Populations: 2017. http://www.smru.st-andrews.ac.uk/research-policy/scos/ [20 Aug 2018]

Russell, D.J.F., Morris, C., Duck, C., Thompson, D. \& Hiby, L. (Submitted). Monitoring long-term changes in UK grey seal Halichoerus grypus pup production. Submitted to this issue of Aquatic Conservation.

Russell, D.J.F., Duck, C., Morris, C. \& Thompson, D. (2016). Independent estimates of grey seal population size: 2008 and 2014. SCOS Briefing paper 16/3, Sea Mammal Research Unit, University of St Andrews. http://www.smru.st-andrews.ac.uk/research-policy/scos/ [20 Aug 2018] 
Russell, D. J. F., McConnell, B., Thompson, D., Duck, C., Morris, C., Harwood, J., \& Matthiopoulos, J. (2013). Uncovering the links between foraging and breeding regions in a highly mobile mammal. Journal of Applied Ecology, 50(2), 499-509.

Russell D.J.F., Hanson N., \& Thomas L. (2016). Marine Strategy Framework Directive: Estimating the European Grey Seal population. Special Committee on Seals 16/09. http://www.smru.standrews.ac.uk/research-policy/scos/ [20 Aug 2018]

Ruxton, G.D. \& Rohani, P. (1998). Fitness-dependent dispersal in metapopulations and its consequences for persistence and synchrony. Journal of Animal Ecology, 67, 530-539.

Smout, S., King, R., \& Pomeroy, P. (Submitted). Environment-sensitive mass changes influence breeding in a marine top predator. Journal of Animal Ecology.

Smout, S., Rindorf, A., Hammond, P., Harwood, J., \& Matthiopoulos, J. (2014). Modelling prey consumption by UK grey seals. ICES Journal of Marine Science, 71(1), 81-89.

Special Committee on Seals (2017). Scientific Advice on Matters Related to the Management of Seal Populations: 2017. Sea Mammal Research Unit, University of St Andrews. Downloaded from http://www.smru.st-andrews.ac.uk/research-policy/scos/

Summers, C.F. (1978). Trends in the size of the British grey seal populations. Journal of Applied Ecology, $15,395-400$.

Thomas, L. (2009). Grey seals red in tooth and claw: how Darwin helps model their population. Significance, 6(3),108-112

Thomas, L. (2010). Estimating the size of the UK grey seal population between 1984 and 2009. SCOS Briefing Paper 11/02. http://www.smru.st-andrews.ac.uk/research-policy/scos/ [20 Aug 2018] 
Thomas, L. (2013). Estimating the size of the UK grey seal population between 1984 and 2012, using established and draft revised priors. SCOS Briefing Paper 13/02. http://www.smru.standrews.ac.uk/research-policy/scos/ [20 Aug 2018]

Thomas, L. (2015). Estimating the size of the UK grey seal population between 1984 and 2014. SCOS Briefing Paper 15/02. http://www.smru.st-andrews.ac.uk/research-policy/scos/ [20 Aug 2018]

Thomas, L., \& Harwood, J. (2008). Estimating the size of the UK grey seal population between 1984 and 2007. SCOS Briefing paper 08/02, Sea Mammal Research Unit, University of St Andrews. http://www.smru.st-andrews.ac.uk/research-policy/scos/ [20 Aug 2018]

Thomas, L. \& Harwood, J. (2003). Estimating grey seal population size using a Bayesian state-space model. SCOS Briefing paper 03/3, Sea Mammal Research Unit, University of St Andrews. http://www.smru.st-andrews.ac.uk/research-policy/scos/ [20 Aug 2018]

Thomas, L., \& Harwood, J. (2009). Estimating the size of the UK grey seal population between 1984 and 2008. SCOS Briefing paper 09/02, Sea Mammal Research Unit, University of St Andrews. http://www.smru.st-andrews.ac.uk/research-policy/scos/ [20 Aug 2018]

Thomas, L., Buckland, S.T. , Newman, K.B. , \& Harwood, J. (2005). A unified framework for modelling wildlife population dynamics. Australian and New Zealand Journal of Statistics, 47, 19-34.

Thomas, L., Burnham, K.P., \& Buckland, S.T. (2004). Temporal inferences from distance sampling surveys. Chapter 5 in Buckland, S.T., D.R. Anderson, K.P. Burnham, J.L. Laake, D.L. Borchers \& L. Thomas (Eds.), Advanced Distance Sampling. Oxford, UK: Oxford University Press.

Thomas, L., Hammill, M.O., \& Bowen, W.D. (2011). Estimated size of the Northwest Atlantic grey seal population 1977-2007. Canadian Science Advisory Secretariat Research Document 2007/082. Canada: Department of Fisheries and Oceans. 
Thompson, D., Duck, C., Morris, C., \& Russell, D. J. F. (Submitted). The status of harbour seals in the United Kingdom. Submitted to this issue of Aquatic Conservation.

Trijoulet, V., Holmes, S. J., \& Cook, R. M. (2017). Grey seal predation mortality on three depleted stocks in the West of Scotland: What are the implications for stock assessments? Canadian Journal of Fisheries and Aquatic Sciences, 75(5), 723-732.

Turchin, P. (2003). Complex population dynamics; a theoretical/empirical synthesis. Princeton, USA: Princeton University Press.

Wyile, O. G. (1988). Assessment of grey seal pup production from counts of pups. PhD Thesis, University of Cambridge. 
Table 1. Prior parameter distributions and summary of posterior distributions. Be and Ga denote beta and gamma distributions, respectively; NS, $\mathrm{IH}, \mathrm{OH}$ and Ork denote North Sea, Inner Hebrides, Outer Hebrides and Orkney regions; dens. dep. is density dependent; c. capacity is carrying capacity.

\begin{tabular}{|l|l|l|l|l|}
\hline Parameter & Prior distribution & Prior mean & Posterior & Prior- \\
& & $(\mathrm{SD})$ & mean (SD) & posterior \\
overlap \\
\hline adult survival $\phi_{a}$ & $0.8+0.17 * \mathrm{Be}(1.6,1.2)$ & $0.90(0.04)$ & $0.95(0.01)$ & $35 \%$ \\
\hline pup survival $\phi_{p \max }$ & $\mathrm{Be}(2.87,1.78)$ & $0.62(0.20)$ & $0.48(0.09)$ & $40 \%$ \\
\hline fecundity $\alpha$ & $0.6+0.4 * \mathrm{Be}(2,1.5)$ & $0.83(0.09)$ & $0.90(0.06)$ & $70 \%$ \\
\hline dens. dep. shape $\rho$ & $\mathrm{Ga}(4,2.5)$ & $10(5)$ & $5.95(1.73)$ & $50 \%$ \\
\hline NS pups at c. capacity $\chi_{1}$ & $\mathrm{Ga}(4,5000)$ & $20000(10000)$ & $15500(8210)$ & $77 \%$ \\
\hline IH pups at c. capacity $\chi_{2}$ & $\mathrm{Ga}(4,1250)$ & $5000(2500)$ & $3110(173)$ & $11 \%$ \\
\hline OH pups at c. capacity $\chi_{3}$ & $\mathrm{Ga}(4,3750)$ & $15000(7500)$ & $11700(535)$ & $14 \%$ \\
\hline Ork pups at c. capacity $\chi_{4}$ & $\mathrm{Ga}(4,10000)$ & $40000(20000)$ & $17800(1680)$ & $9 \%$ \\
\hline observation precision $\psi$ & $\mathrm{Ga}(2.1,66.67)$ & $140(96.61)$ & $112(34.60)$ & $49 \%$ \\
\hline sex ratio $\omega$ & $1.6+\mathrm{Ga}(28.08,3.70 \mathrm{E}-3)$ & $1.7(0.02)$ & $1.7(0.02)$ & $99 \%$ \\
\hline
\end{tabular}

1040 


\section{Figure Legends}

1043 Figure 1. Posterior mean estimates of regional pup production (solid lines) from the state-space 1044 model, with 95\% credible intervals (dashed lines). Thick red lines show results from model fitted to pup production plus the total population estimate of 2008; thinner blue lines show the fit to pup production alone. The two sets of lines are nearly identical, so the blue lines are partly hidden. Circles show pup production data.

Figure 2. Posterior mean estimates of adult population size in annually monitored colonies (solid lines) from the state-space model, with 95\% credible intervals (dashed lines). Thick red lines show results from model fitted to pup production plus the total population estimate of 2008; thinner blue lines

1051 show results from fit to pup production alone. Circle shows the (scaled) total population estimate of 2008 , with vertical lines indicating $95 \%$ probability interval on the estimate.

1053

Figure 3. Posterior (histograms) and prior (solid lines) parameter distributions for the state-space model fitted to pup production plus the total population estimate of 2008 . The vertical dashed line shows the posterior mean; its value is also given in the title of each plot below the parameter name, with the associated standard deviation in parentheses. 
Table 1. Prior parameter distributions and summary of posterior distributions. Be and Ga denote beta and gamma distributions, respectively; NS, $\mathrm{IH}, \mathrm{OH}$ and Ork denote North Sea, Inner Hebrides, Outer Hebrides and Orkney regions; dens. dep. is density dependent; c. capacity is carrying capacity.

\begin{tabular}{|l|l|l|l|l|}
\hline Parameter & Prior distribution & $\begin{array}{l}\text { Prior mean } \\
\text { (SD) }\end{array}$ & $\begin{array}{l}\text { Posterior } \\
\text { mean (SD) }\end{array}$ & $\begin{array}{l}\text { Prior- } \\
\text { posterior } \\
\text { overlap }\end{array}$ \\
\hline adult survival $\phi_{a}$ & $0.8+0.17^{*} \mathrm{Be}(1.6,1.2)$ & $0.90(0.04)$ & $0.95(0.01)$ & $35 \%$ \\
\hline pup survival $\phi_{p \max }$ & $\mathrm{Be}(2.87,1.78)$ & $0.62(0.20)$ & $0.48(0.09)$ & $40 \%$ \\
\hline fecundity $\alpha$ & $0.6+0.4^{*} \mathrm{Be}(2,1.5)$ & $0.83(0.09)$ & $0.90(0.06)$ & $70 \%$ \\
\hline dens. dep. shape $\rho$ & $\mathrm{Ga}(4,2.5)$ & $10(5)$ & $5.95(1.73)$ & $50 \%$ \\
\hline $\mathrm{NS}$ pups at c. capacity $\chi_{1}$ & $\mathrm{Ga}(4,5000)$ & $20000(10000)$ & $15500(8210)$ & $77 \%$ \\
\hline IH pups at c. capacity $\chi_{2}$ & $\mathrm{Ga}(4,1250)$ & $5000(2500)$ & $3110(173)$ & $11 \%$ \\
\hline OH pups at c. capacity $\chi_{3}$ & $\mathrm{Ga}(4,3750)$ & $15000(7500)$ & $11700(535)$ & $14 \%$ \\
\hline Ork pups at c. capacity $\chi_{4}$ & $\mathrm{Ga}(4,10000)$ & $40000(20000)$ & $17800(1680)$ & $9 \%$ \\
\hline observation precision $\psi$ & $\mathrm{Ga}(2.1,66.67)$ & $140(96.61)$ & $112(34.60)$ & $49 \%$ \\
\hline sex ratio $\omega$ & $1.6+\mathrm{Ga}(28.08,3.70 \mathrm{E}-3)$ & $1.7(0.02)$ & $1.7(0.02)$ & $99 \%$ \\
\hline
\end{tabular}


North Sea

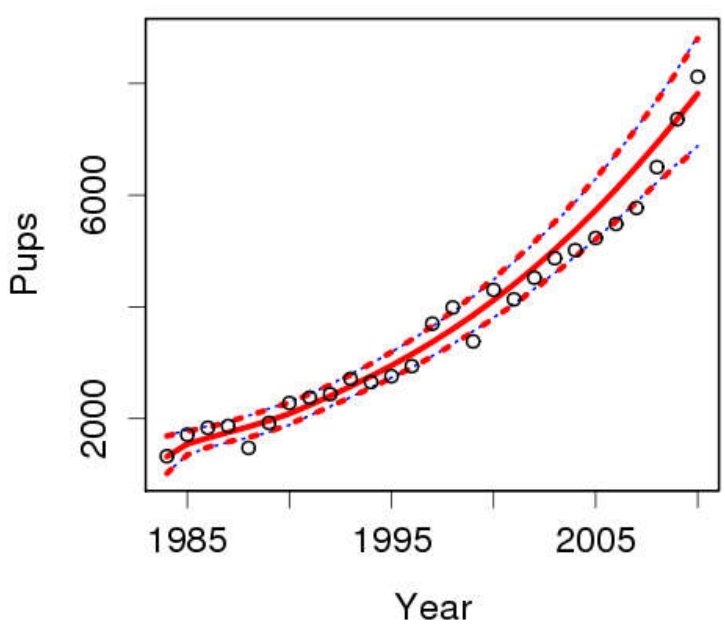

Outer Hebrides

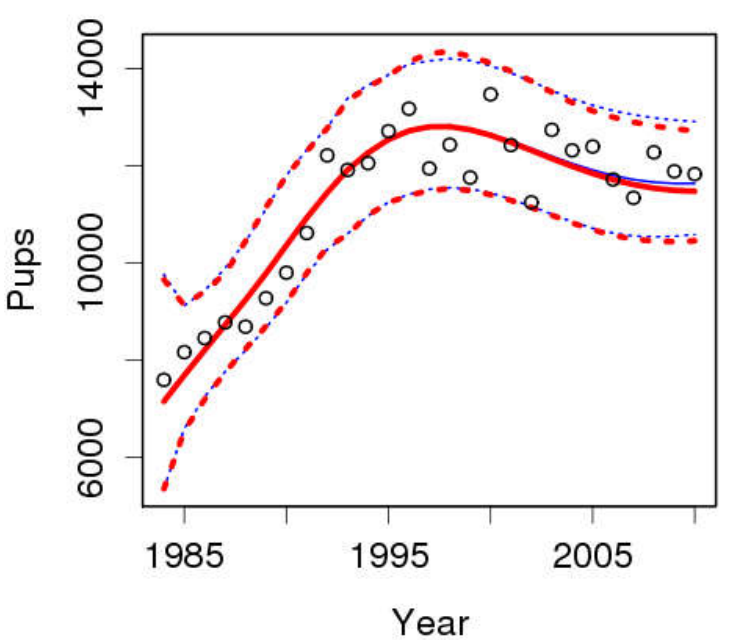

Inner Hebrides

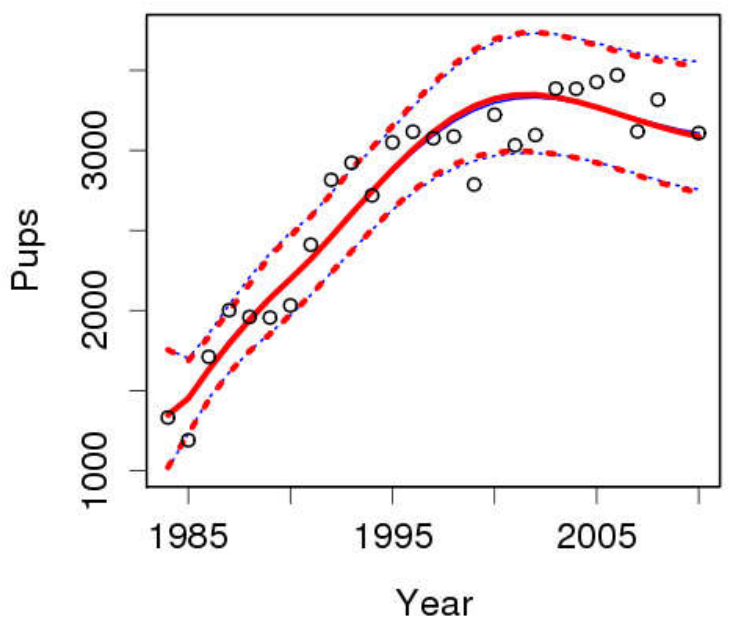

\section{Orkney}

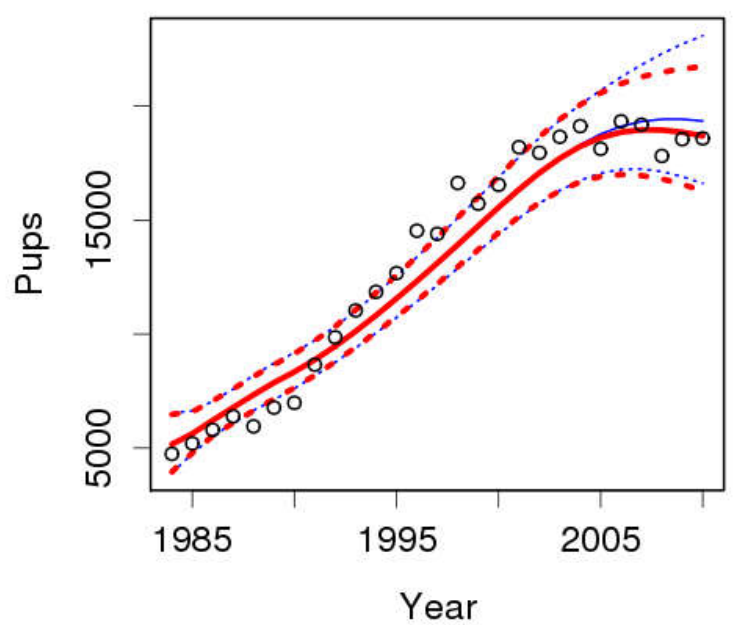

Figure 1. Posterior mean estimates of regional pup production (solid lines) from the state-space model, with $95 \%$ credible intervals (dashed lines). Thick red lines show results from model fitted to pup production plus the total population estimate of 2008; thinner blue lines show the fit to pup production alone. The two sets of lines are nearly identical, so the blue lines are partly hidden. Circles show pup production data. 


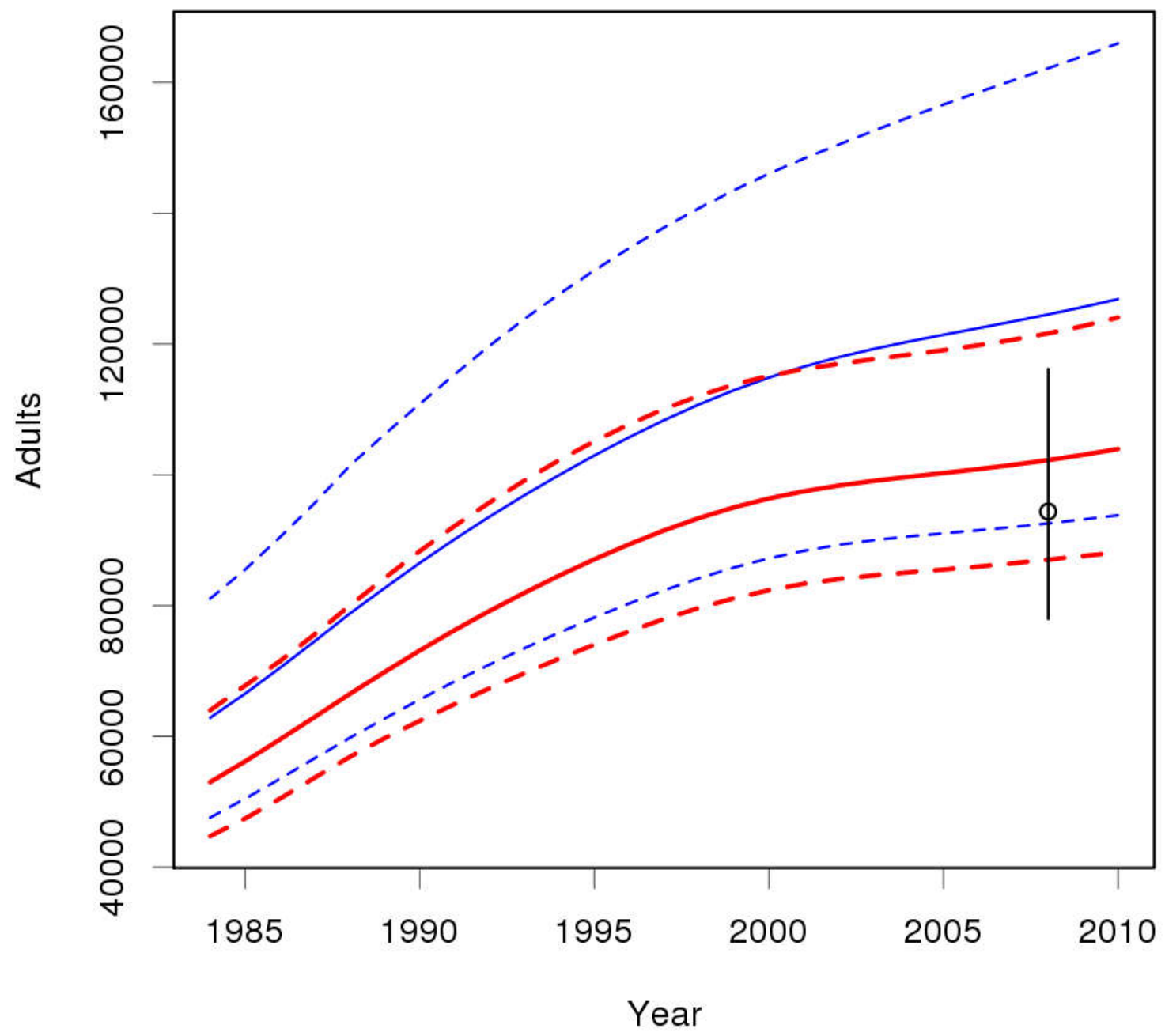

Figure 2. Posterior mean estimates of adult population size in annually monitored colonies (solid lines) from the state-space model, with $95 \%$ credible intervals (dashed lines). Thick red lines show results from model fitted to pup production plus the total population estimate of 2008; thinner blue lines show results from fit to pup production alone. Circle shows the (scaled) total population estimate of 2008 , with vertical lines indicating $95 \%$ probability interval on the estimate. 

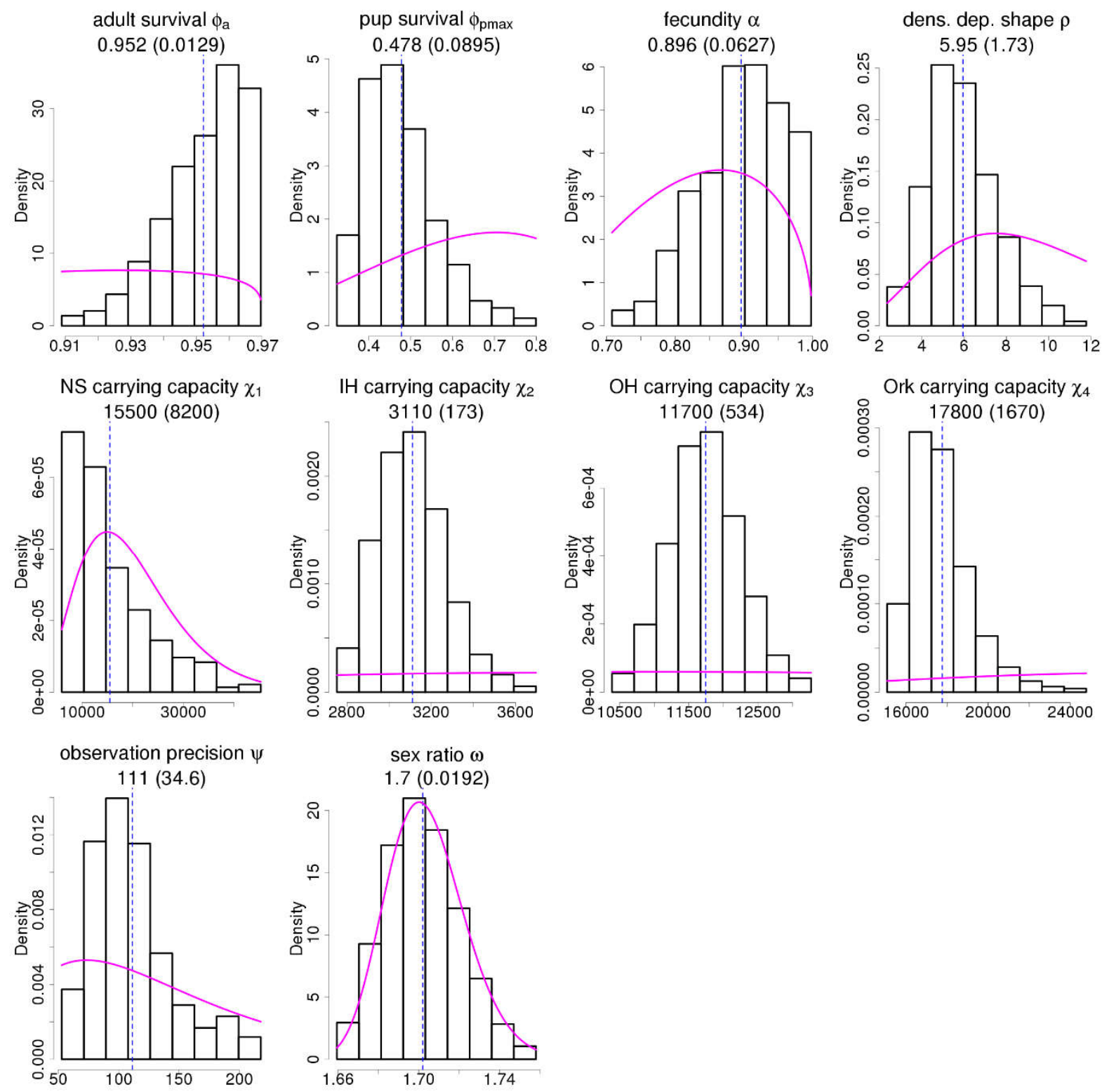

Figure 3. Posterior (histograms) and prior (solid lines) parameter distributions for the state-space model fitted to pup production plus the total population estimate of 2008. The vertical dashed line shows the posterior mean; its value is also given in the title of each plot below the parameter name, with the associated standard deviation in parentheses. 
1 Supporting Information for "Modelling the population size and 2 dynamics of the British grey seal"

3 Len Thomas, Debbie J.F. Russell, Callan Duck, Chris D. Morris, Mike Lonergan, Fanny Empacher, Dave 4 Thompson and John Harwood.

$5 \quad$ S1. Supplementary figures and tables

6

7 Table S1. Pup production estimates used in the population dynamics model. NA denotes missing data.

\begin{tabular}{|l|l|l|l|l|l|}
\hline Year & North Sea & Inner Hebrides & Outer Hebrides & Orkney & Total \\
\hline 1984 & 1325 & 1332 & 7594 & 4741 & 14992 \\
\hline 1985 & 1711 & 1190 & 8165 & 5199 & 16265 \\
\hline 1986 & 1834 & 1711 & 8455 & 5796 & 17796 \\
\hline 1987 & 1867 & 2002 & 8777 & 6389 & 19035 \\
\hline 1988 & 1474 & 1960 & 8689 & 5948 & 18071 \\
\hline 1989 & 1922 & 1956 & 9275 & 6773 & 19926 \\
\hline 1990 & 2278 & 2032 & 9801 & 6982 & 21093 \\
\hline 1991 & 2375 & 2411 & 10617 & 8653 & 24056 \\
\hline 1992 & 2436 & 2816 & 12215 & 9854 & 27321 \\
\hline 1993 & 2710 & 2923 & 11915 & 11034 & 28582 \\
\hline 1994 & 2652 & 2719 & 12054 & 11851 & 29276 \\
\hline 1995 & 2757 & 3050 & 12713 & 12670 & 31190 \\
\hline 1996 & 2938 & 3117 & 13176 & 14531 & 33762 \\
\hline 1997 & 3698 & 3076 & 11946 & 14395 & 33115 \\
\hline 1998 & 3989 & 3087 & 12434 & 16625 & 36135 \\
\hline 1999 & 3380 & 2787 & 11759 & 15720 & 33646 \\
\hline 2000 & 4303 & 3223 & 13472 & 16546 & 37544 \\
\hline 2001 & 4134 & 3032 & 12427 & 18196 & 37789 \\
\hline 2002 & 4520 & 3096 & 11248 & 17952 & 36816 \\
\hline 2003 & 4870 & 3386 & 12741 & 18652 & 39649 \\
\hline 2004 & 5015 & 3385 & 12319 & 19123 & 39842 \\
\hline 2005 & 5232 & 3427 & 12397 & 18126 & 39182 \\
\hline 2006 & 5484 & 3470 & 11719 & 19332 & 40005 \\
\hline 2007 & 5771 & 3118 & 11342 & 19184 & 39415 \\
\hline 2008 & 6501 & 3317 & 12279 & 17813 & 39910 \\
\hline 2009 & 7360 & NA & 11887 & 18548 & NA \\
\hline 2010 & 8119 & 3108 & 11831 & 18582 & 41640 \\
\hline & & & & & \\
\hline
\end{tabular}


Table S2. Estimates of population size of seals associated with regularly-monitored colonies, in thousands, at the beginning of each breeding season from 1984-2010, made using the model of British grey seal population dynamics fitted to pup production estimates from 1984-2010 and the total population estimate from 2008 . Numbers are posterior means followed by $95 \%$ credible intervals in brackets. The last column is the ratio of estimated total population size in each year and that in the previous year.

\begin{tabular}{|c|c|c|c|c|c|c|}
\hline Year & North Sea & Inner Hebrides & Outer Hebrides & Orkney & Total & Growth rate \\
\hline 1984 & $4.8(45.9)$ & $5.1(4.26 .3)$ & 23.9 (19.7 29.6) & $19.2(15.923 .5)$ & $53(44.764)$ & - \\
\hline 1985 & $5.1(4.36 .2)$ & $5.4(4.46 .6)$ & $25.3(20.831 .3)$ & $20.4(1724.8)$ & $56.2(47.467 .8)$ & $1.06(1.041 .1)$ \\
\hline 1986 & $5.5(4.66 .6)$ & $5.7(4.76 .9)$ & 26.7 (22.1 32.9) & $21.7(18.226 .3)$ & 59.5 (50.5 71.5) & $1.06(1.031 .1)$ \\
\hline 1987 & $5.9(57.1)$ & $6(57.3)$ & $28(23.334 .4)$ & 23.1 (19.5 28) & $63(53.775 .6)$ & $1.06(1.031 .1)$ \\
\hline 1988 & $6.3(5.37 .6)$ & $6.4(5.37 .7)$ & $29.1(24.235 .8)$ & 24.7 (20.9 30) & 66.5 (56.9 80) & 1.06 (1.03 1.1) \\
\hline 1989 & $6.7(5.78 .2)$ & $6.7(5.68 .2)$ & $30(24.836 .8)$ & $26.4(22.332)$ & $69.9(59.784 .2)$ & 1.05 (1.03 1.1) \\
\hline 1990 & $7.2(6.18 .7)$ & $7(5.98 .6)$ & 30.7 (25.3 37.7) & $28.2(23.934 .2)$ & $73.1(62.488 .3)$ & 1.05 (1.03 1.1) \\
\hline 1991 & $7.7(6.59 .3)$ & $7.3(6.19)$ & $31.1(25.638 .3)$ & $30.1(25.536 .4)$ & 76.2 (64.9 92.2) & $1.04(1.031 .1)$ \\
\hline 1992 & 8.2 (7 10) & $7.6(6.39 .3)$ & $31.3(25.738 .7)$ & 32 (27.1 38.7) & $79.2(67.395 .8)$ & $1.04(1.031 .1)$ \\
\hline 1993 & $8.8(7.4$ 10.7) & $7.8(6.59 .6)$ & 31.3 (25.8 38.7) & $34(28.841 .1)$ & 82 (69.7 99.1) & $1.04(1.031)$ \\
\hline 1994 & $9.4(7.911 .4)$ & $8(6.69 .9)$ & $31.2(25.738 .5)$ & $36(30.443 .5)$ & 84.6 (71.9 102.2) & $1.03(1.021)$ \\
\hline 1995 & $10.1(8.512 .2)$ & 8.1 (6.7 10.1) & $30.9(25.538 .1)$ & $38(32.145 .8)$ & 87.1 (74 105.1) & $1.03(1.021)$ \\
\hline 1996 & $10.8(9.1$ 13.1) & 8.2 (6.7 10.2) & $30.5(25.237 .5)$ & $39.9(33.648 .2)$ & 89.4 (76.1 107.7) & $1.03(1.021)$ \\
\hline 1997 & 11.5 (9.7 14) & 8.2 (6.7 10.2) & 30.1 (25 36.9) & 41.7 (35 50.3) & 91.5 (78 110.1) & $1.02(1.011)$ \\
\hline 1998 & $12.3(10.3$ 14.9) & 8.1 (6.7 10.1) & $29.7(24.736 .3)$ & $43.3(36.352 .3)$ & 93.4 (79.7 112.1) & $1.02(1.011)$ \\
\hline 1999 & 13.1 (11 15.9) & $8.1(6.610)$ & $29.3(24.435 .7)$ & 44.5 (37.3 53.8) & $95(81.1113 .8)$ & $1.02(1.011)$ \\
\hline 2000 & 14 (11.7 17) & $8(6.69 .9)$ & $28.9(24.235 .2)$ & 45.5 (37.9 54.9) & 96.4 (82.4 115.1) & $1.01(11)$ \\
\hline 2001 & $14.9(12.4$ 18.1) & 7.9 (6.5 9.7) & $28.6(23.934 .7)$ & 46.1 (38.3 55.6) & 97.5 (83.3 116.2) & $1.01(11)$ \\
\hline 2002 & $15.9(13.2$ 19.3) & $7.8(6.49 .6)$ & $28.4(23.734 .4)$ & $46.3(38.456)$ & $98.4(84.1117)$ & $1.01(0.991)$ \\
\hline 2003 & 16.9 (14 20.5) & $7.7(6.49 .4)$ & $28.2(23.634 .1)$ & $46.3(38.256)$ & 99.1 (84.6 117.7) & $1.01(0.991)$ \\
\hline 2004 & $18(14.821 .8)$ & $7.6(6.39 .3)$ & $28.1(23.534)$ & 46 (37.9 55.8) & $99.7(85.1118 .4)$ & 1.01 (0.99 1) \\
\hline 2005 & 19.1 (15.6 23.2) & $7.5(6.29 .2)$ & $28(23.434)$ & $45.6(37.455 .6)$ & $100.3(85.5$ 119.1) & $1.01(0.991)$ \\
\hline 2006 & $20.2(16.324 .8)$ & $7.5(6.29 .2)$ & $28.1(23.434 .1)$ & $45.1(36.855 .3)$ & 100.9 (86 119.8) & 1.01 (0.99 1) \\
\hline 2007 & $21.4(16.826 .4)$ & $7.4(6.2$ 9.1) & 28.1 (23.4 34.3) & $44.6(36.255 .1)$ & 101.5 (86.5 120.7) & $1.01(0.991)$ \\
\hline 2008 & 22.5 (17 28.2) & 7.4 (6.1 9.1) & $28.2(23.534 .5)$ & $44.1(35.654 .9)$ & $102.2(87121.6)$ & $1.01(0.991)$ \\
\hline 2009 & $23.7(17.130 .1)$ & 7.4 (6.1 9.1) & $28.4(23.634 .8)$ & $43.6(3554.6)$ & 103.1 (87.6 122.8) & $1.01(0.991)$ \\
\hline 2010 & $24.8(17.132 .1)$ & $7.4(6.2$ 9.1) & $28.5(23.635 .1)$ & 43.1 (34.6 54.4) & 104 (88.1 124.1) & 1.01 (0.99 1) \\
\hline
\end{tabular}


Table S3. Population age structure estimated using posterior mean parameter estimates and assuming 17 deterministic population dynamics. Left column shows stable age structure during initial exponential 18 stage of population growth; right column shows stable age structure at carrying capacity. Age 0 (pups) 19 are both males and females; ages 1 and higher are females.

\begin{tabular}{|l|l|l|}
\hline & \multicolumn{2}{c|}{ Proportion of population at this age } \\
\hline \multicolumn{1}{|c|}{ Age } & During exponential growth & At carrying capacity \\
\hline 0 (male and female pups) & 0.332 & 0.412 \\
\hline 1 (females) & 0.074 & 0.029 \\
\hline 2 (females) & 0.066 & 0.027 \\
\hline 3 (females) & 0.059 & 0.025 \\
\hline 4 (females) & 0.052 & 0.024 \\
\hline 5 (females) & 0.046 & 0.023 \\
\hline $6+$ (females) & 0.371 & 0.460 \\
\hline
\end{tabular}


Table S4. Survival data used to inform the survival and sex ratio priors. CMR refers to Capture-Mark-Recapture studies and can be based on brands (permanent but can be misidentified), passive tagging (can be lost or misidentified), active tagging (can be lost), Photo-ID (can be misidentified). Except for active tagging, estimates of survival depend on the accuracy of re-sighting probabilities and, if appropriate, tag loss. If sex-specific sample sizes are not reported then total $n$ is given.

\begin{tabular}{|c|c|c|c|c|c|c|c|c|c|c|c|c|}
\hline \multirow{2}{*}{$\begin{array}{l}\text { Age } \\
\text { class }\end{array}$} & \multicolumn{3}{|c|}{ females } & \multicolumn{3}{|c|}{ males } & \multirow{2}{*}{$\begin{array}{c}\text { Total } \\
\mathbf{n}\end{array}$} & \multirow{2}{*}{$\begin{array}{l}\text { Time } \\
\text { period }\end{array}$} & \multirow{2}{*}{ Data } & \multirow{2}{*}{ Location } & \multirow{2}{*}{ Considerations } & \multirow{2}{*}{ Source } \\
\hline & mean & uncertainty & n & mean & uncertainty & n & & & & & & \\
\hline Pup & 0.66 & & 1036 & 0.66 & & 294 & & $\begin{array}{l}1972 \\
1975\end{array}$ & $\begin{array}{l}\text { Aged shot } \\
\text { individuals }\end{array}$ & $\begin{array}{l}\text { Farne } \\
\text { Islands, } \\
\text { UK }\end{array}$ & $\begin{array}{l}\text { Accounted for effect } \\
\text { of previous culls on } \\
\text { sample structure. } \\
\text { Based on life tables. }\end{array}$ & $\begin{array}{l}\text { Harwood \& Prime } \\
1978\end{array}$ \\
\hline Pup & 0.65 & $\begin{array}{l}95 \% \text { Cls: } \\
0.39-0.85\end{array}$ & 180 & 0.50 & $\begin{array}{l}95 \% \mathrm{Cls}: \\
0.25-0.75\end{array}$ & 182 & & $\begin{array}{l}1997- \\
1999\end{array}$ & $\begin{array}{l}\text { CMR (hat } \\
\text { tag) }\end{array}$ & $\begin{array}{l}\text { Isle of } \\
\text { May and } \\
\text { Farne } \\
\text { Islands, } \\
\text { UK }\end{array}$ & $\begin{array}{l}\text { Tag loss accounted } \\
\text { for. Telemetry data } \\
\text { used to inform re- } \\
\text { sighting probability }\end{array}$ & $\begin{array}{l}\text { Reanalysis of data } \\
\text { from Hall, McConnell } \\
\text { \& Barker 2001; Hall, } \\
\text { McConnell \& Barker } \\
\text { 2002; grey pup seal } \\
\text { telemetry data } \\
\text { (Carter et al., 2017) }\end{array}$ \\
\hline Pup & 0.54 & $\begin{array}{l}95 \% \text { Cls: } \\
0.18-0.86\end{array}$ & 27 & 0.43 & $\begin{array}{l}95 \% \text { Cls: } \\
0.11-0.82\end{array}$ & 28 & & 2002 & $\begin{array}{l}\text { CMR } \\
\text { (telemetry } \\
\text { data) }\end{array}$ & $\begin{array}{l}\text { Isle of } \\
\text { May, UK }\end{array}$ & Tag loss accounted for & $\begin{array}{l}\text { Reanalysis of data } \\
\text { from Hall, Thomas \& } \\
\text { McConnell } 2009\end{array}$ \\
\hline Pup & $\begin{array}{l}0.76 \\
0.55\end{array}$ & & & $\begin{array}{l}0.38 \\
0.53\end{array}$ & & & $\begin{array}{l}1185 \\
2295\end{array}$ & $\begin{array}{l}2000- \\
2004 \\
2005- \\
2009\end{array}$ & $\begin{array}{l}\text { Aged shot } \\
\text { individuals }\end{array}$ & Baltic & $\begin{array}{l}\text { Samples assumed } \\
\text { representative. Based } \\
\text { on life tables }\end{array}$ & $\begin{array}{l}\text { Kauhala, Ahola \& } \\
\text { Kunnasranta } 2012\end{array}$ \\
\hline$\leq 4$ & $\begin{array}{l}0.735 \\
0.331\end{array}$ & $\begin{array}{l}S E=0.016 \\
S E=0.024\end{array}$ & $\begin{array}{l}1700 \\
1182\end{array}$ & & & & & $\begin{array}{l}1985- \\
1989 \\
1998- \\
2002\end{array}$ & $\begin{array}{l}\text { CMR } \\
\text { (brand) }\end{array}$ & $\begin{array}{l}\text { Sable } \\
\text { Island, } \\
\text { Canada }\end{array}$ & $\begin{array}{l}\text { Includes the data } \\
\text { from Schwarz \& Stobo } \\
(2000)\end{array}$ & $\begin{array}{l}\text { den Heyer, Bowen \& } \\
\text { Mcmillan } 2014\end{array}$ \\
\hline Adult & 0.95 & & 239 & & & & & $\begin{array}{l}1956- \\
1966\end{array}$ & $\begin{array}{l}\text { Aged shot } \\
\text { individuals }\end{array}$ & UK & $\begin{array}{l}\text { Samples assumed } \\
\text { representative. Based } \\
\text { on life tables }\end{array}$ & $\begin{array}{l}\text { Data from Hewer } \\
\text { 1974, analysed by } \\
\text { Lonergan } 2012\end{array}$ \\
\hline
\end{tabular}




\begin{tabular}{|c|c|c|c|c|c|c|c|c|c|c|c|}
\hline$\geq 10$ & & & & 0.80 & & 294 & $\begin{array}{l}1972 \\
1975\end{array}$ & $\begin{array}{l}\text { Aged shot } \\
\text { individuals }\end{array}$ & $\begin{array}{l}\text { Farne } \\
\text { Islands, } \\
\text { UK }\end{array}$ & $\begin{array}{l}\text { Accounted for } \\
\text { population trajectory. } \\
\text { Assumed samples are } \\
\text { representative within } \\
\text { focal age class. }\end{array}$ & $\begin{array}{l}\text { Harwood \& Prime } \\
1978\end{array}$ \\
\hline$\geq 7$ & $\begin{array}{l}0.935 \\
(0.90- \\
0.96)\end{array}$ & & 1036 & & & & $\begin{array}{l}1972 \\
1975\end{array}$ & $\begin{array}{l}\text { Aged shot } \\
\text { individuals }\end{array}$ & $\begin{array}{l}\text { Farne } \\
\text { Islands, } \\
\text { UK }\end{array}$ & As above & $\begin{array}{l}\text { Harwood \& Prime } \\
1978 \text { (reanalysed by } \\
\text { Lonergan 2012) }\end{array}$ \\
\hline Adult & 0.941 & $\begin{array}{l}95 \% \mathrm{Cls}: \\
0.929- \\
0.954\end{array}$ & 273 & & & & $\begin{array}{l}1987- \\
2014\end{array}$ & $\begin{array}{l}\text { CMR } \\
\text { (brand, } \\
\text { flipper tag, } \\
\text { photo ID) }\end{array}$ & $\begin{array}{l}\text { Isle of } \\
\text { May }\end{array}$ & $\begin{array}{l}\text { Tag loss and } \\
\text { differential sighting } \\
\text { probability accounted } \\
\text { for. Survival } \\
\text { confounded with } \\
\text { permanent } \\
\text { emigration }\end{array}$ & $\begin{array}{l}\text { Smout, King \& } \\
\text { Pomeroy, Submitted }\end{array}$ \\
\hline Adult & 0.886 & $\begin{array}{l}95 \% \mathrm{Cls}: \\
0.871- \\
0.900\end{array}$ & 584 & & & & $\begin{array}{l}1993- \\
2013\end{array}$ & As above & $\begin{array}{l}\text { North } \\
\text { Rona, UK }\end{array}$ & As above & As above \\
\hline$\geq 4$ & 0.976 & $S E=0.001$ & 3178 & & & 1727 & $\begin{array}{l}1969- \\
2002\end{array}$ & $\begin{array}{l}\text { CMR } \\
\text { (brand) }\end{array}$ & $\begin{array}{l}\text { Sable } \\
\text { Island, } \\
\text { Canada }\end{array}$ & $\begin{array}{l}\text { Tagged as pups. } \\
\text { Confounded with } \\
\text { permanent } \\
\text { emigration (rare) }\end{array}$ & $\begin{array}{l}\text { den Heyer \& Bowen } \\
2017\end{array}$ \\
\hline $4-24$ & 0.989 & $S E=0.001$ & $\begin{array}{l}\text { As } \\
\text { above }\end{array}$ & 0.970 & $S E=0.002$ & $\begin{array}{l}\text { As } \\
\text { above }\end{array}$ & $\begin{array}{l}\text { As } \\
\text { above }\end{array}$ & As above & As above & As above & As above \\
\hline$\geq 25$ & 0.904 & $S E=0.004$ & $\begin{array}{l}\text { As } \\
\text { above }\end{array}$ & 0.77 & $S E=0.01$ & $\begin{array}{l}\text { As } \\
\text { above }\end{array}$ & $\begin{array}{l}\text { As } \\
\text { above }\end{array}$ & As above & As above & As above & As above \\
\hline Adult & 0.976 & $S E=0.001$ & $\begin{array}{l}\text { As } \\
\text { above }\end{array}$ & 0.943 & $S E=0.003$ & $\begin{array}{l}\text { As } \\
\text { above }\end{array}$ & $\begin{array}{l}\text { As } \\
\text { above }\end{array}$ & As above) & As above & As above & As above \\
\hline
\end{tabular}


Table S5. Fecundity data used to inform the fecundity priors. CMR refers to Capture-Mark-Recapture studies and can be based on brands (permanent but can be misidentified), passive tagging (can be lost or misidentified), Photo-ID (can be misidentified). Estimates of fecundity depend on the accuracy of re-sighting probabilities and, if appropriate, tag loss.

\begin{tabular}{|c|c|c|c|c|c|c|c|c|}
\hline Rate & Mean & Uncertainty & $\mathbf{n}$ & $\begin{array}{l}\text { Time } \\
\text { period }\end{array}$ & Data & Location & Considerations & Source \\
\hline Pregnancy & 0.93 & & 79 & $\begin{array}{l}1956- \\
1963\end{array}$ & Shot samples & & & Hewer 1964 \\
\hline Pregnancy & 0.94 & $\begin{array}{l}95 \% \text { Cls: } \\
0.89-0.97\end{array}$ & 140 & $\begin{array}{l}1979- \\
1981\end{array}$ & Shot samples & $\begin{array}{l}\text { Farne Islands, } \\
\text { UK }\end{array}$ & & Boyd 1985 \\
\hline Pregnancy & 0.83 & $\begin{array}{l}95 \% \text { Cls: } \\
0.74-0.89\end{array}$ & 88 & 1978 & Shot samples & $\begin{array}{l}\text { Outer Hebrides, } \\
\text { UK }\end{array}$ & & Boyd 1985 \\
\hline Pregnancy & $\begin{array}{l}0.88- \\
1\end{array}$ & & 526 & $\begin{array}{l}1968- \\
1992\end{array}$ & Shot samples & $\begin{array}{l}\text { Sable Island, } \\
\text { Canada }\end{array}$ & Aged $\geq 6$ years old & $\begin{array}{l}\text { Hammill \& Gosselin } \\
1995\end{array}$ \\
\hline Birth & 0.73 & 0.015 & 174 & $\begin{array}{l}1983- \\
2005\end{array}$ & CMR (brand) & $\begin{array}{l}\text { Sable Island, } \\
\text { Canada }\end{array}$ & $\begin{array}{l}\text { Aged } 4-15 \text { years. } \\
\text { Unobserved pupping not considered } \\
\text { (likely rare) }\end{array}$ & Bowen et al. 2006 \\
\hline Birth & 0.83 & 0.034 & 32 & $\begin{array}{l}1983- \\
2005\end{array}$ & As above & As above & $\begin{array}{l}\text { Aged } 16-25 \text { year } \\
\text { Unobserved pupping not considered } \\
\text { (likely rare) }\end{array}$ & As above \\
\hline Birth & 0.57 & 0.03 & 39 & $\begin{array}{l}1983- \\
2005\end{array}$ & As above & As above & $\begin{array}{l}\text { Aged } 26-35 \text { years } \\
\text { Unobserved pupping not considered } \\
\text { (likely rare) }\end{array}$ & As above \\
\hline Birth & 0.790 & $\begin{array}{l}95 \% \text { Cls: } \\
0.766- \\
0.812\end{array}$ & 584 & $\begin{array}{l}1993- \\
2013\end{array}$ & $\begin{array}{l}\text { CMR (brand, flipper tag, } \\
\text { photo ID) }\end{array}$ & North Rona, UK & Accounted for unobserved pupping & $\begin{array}{l}\text { Smout et al. } \\
\text { Submitted }\end{array}$ \\
\hline Birth & 0.816 & $\begin{array}{l}95 \% \text { Cls: } \\
0.787- \\
0.841\end{array}$ & 273 & $\begin{array}{l}1987- \\
2014\end{array}$ & $\begin{array}{l}\text { CMR } \\
\text { (brand, flipper tag, photo } \\
\text { ID) }\end{array}$ & Isle of May, UK & As above & As above \\
\hline Birth & 0.79 & & 1727 & $\begin{array}{l}1992- \\
2002\end{array}$ & CMR (brand) & $\begin{array}{l}\text { Sable Island, } \\
\text { Canada }\end{array}$ & $\begin{array}{l}\text { Estimated transitions: } \\
\text { unobserved to breeder }=0.41-0.64 \text {, } \\
\text { breeder to breeder }=0.76-0.89\end{array}$ & $\begin{array}{l}\text { den Heyer \& Bowen } \\
2017\end{array}$ \\
\hline
\end{tabular}




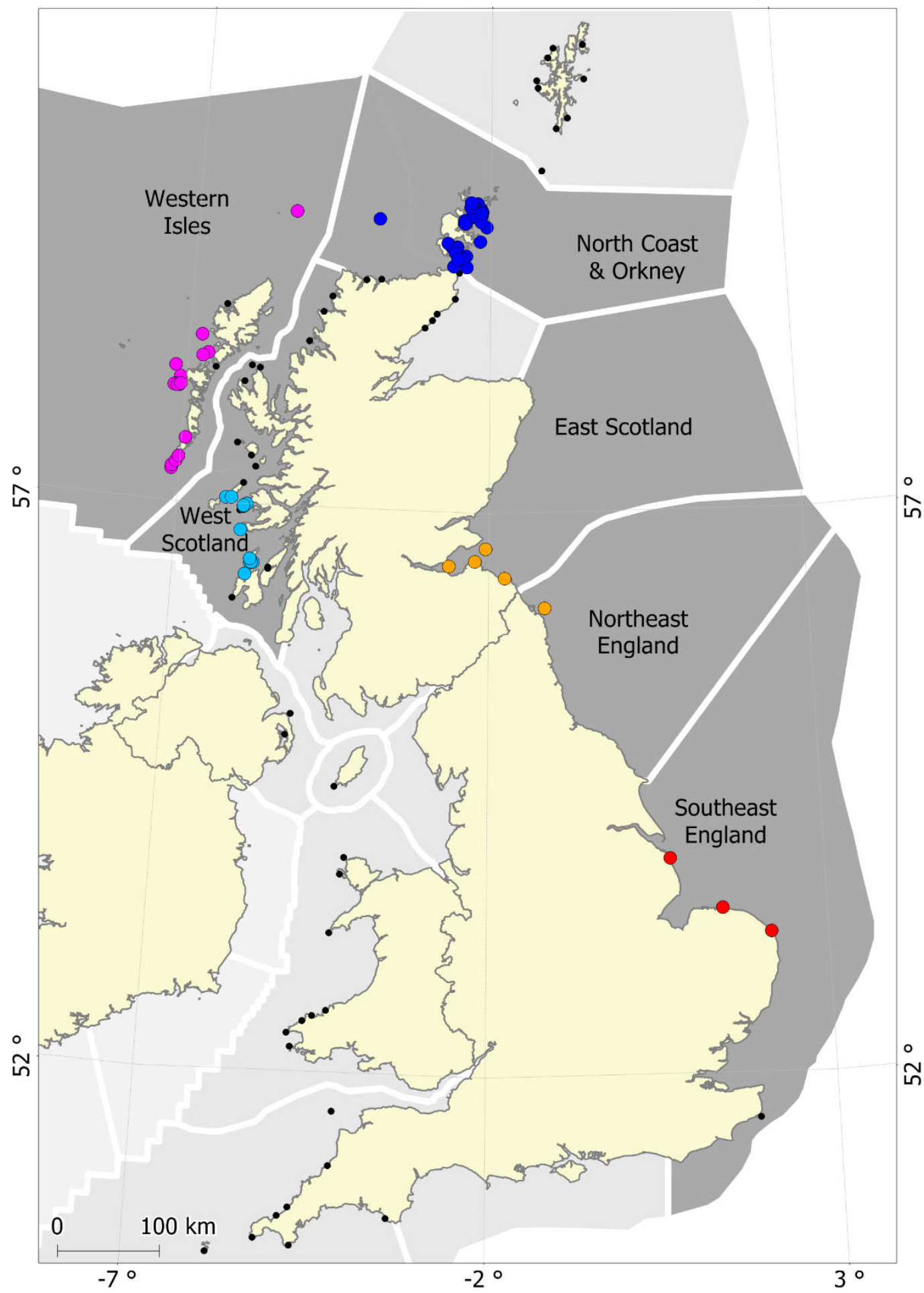

Figure S1. Map showing the location of all grey seal colonies in the UK and Isle of Man. Regularly monitored colonies, which are the ones included in the analysis undertaken in this paper, are colour coded by region: Inner Hebrides (cyan), Outer Hebrides (pink), Orkney (blue) and North Sea (central orange, south - red; these were combined in the analysis reported here). Also shown are the names and boundaries of Seal Management Units associated with these regions. 

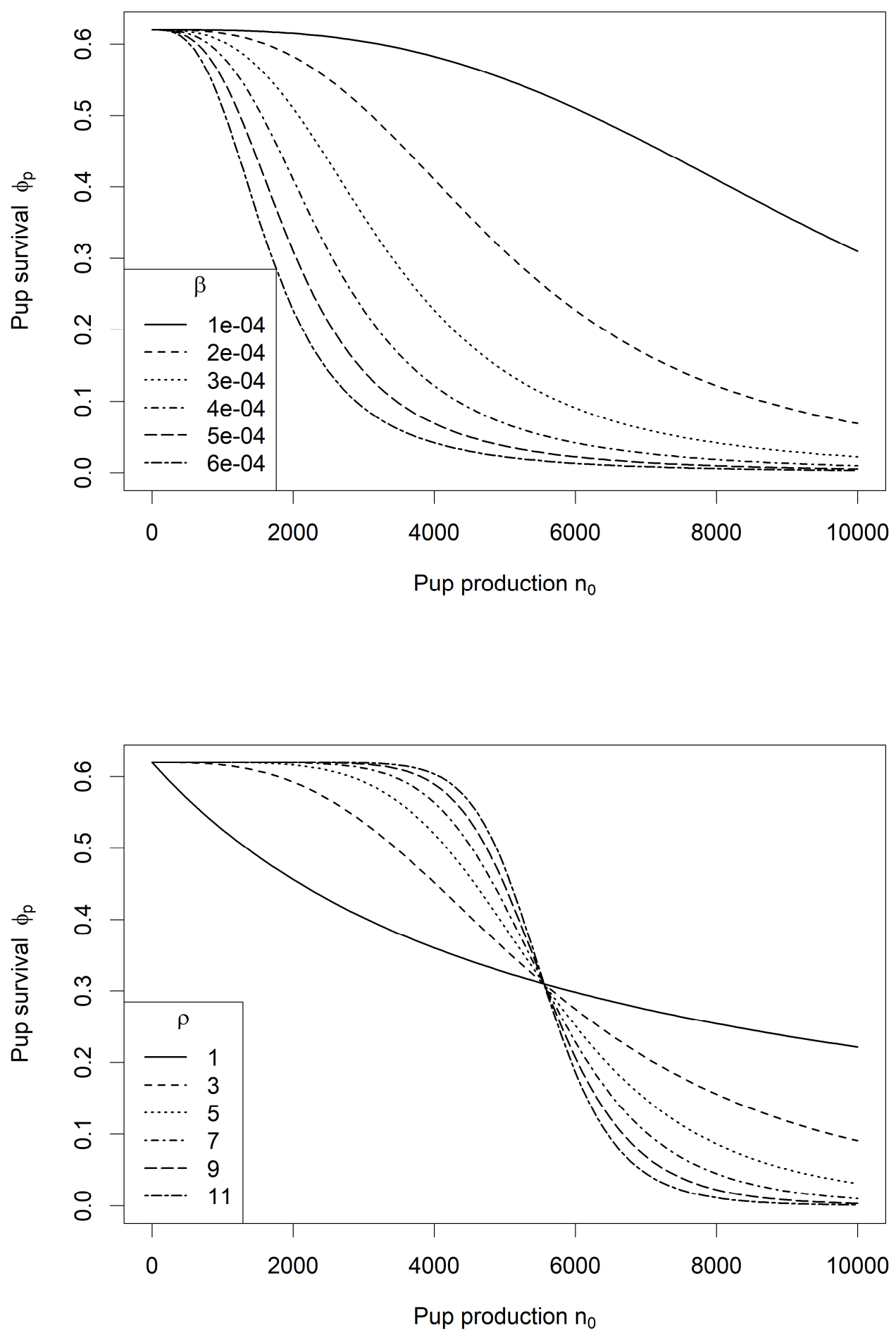

37 Figure S2. Illustration of the effect of the density dependence parameters $\beta$ (rate) and $\rho$ (shape) on 38 the relationship between pup production $n_{0}$ and pup survival $\phi_{p}$. Top panel: $\beta$ varies while $\rho$ is set 39 at 1 ; increasing $\beta$ increases the overall rate at which survival decreases. Bottom panel: $\rho$ varies while $40 \beta$ is set at 0.0018 . Increasing $\rho$ causes survival to remain higher as pup production increases, but then 41 decline more steeply when it does start to decline. In this illustration, all other model parameters are 42 set at their prior means (Table 1). 

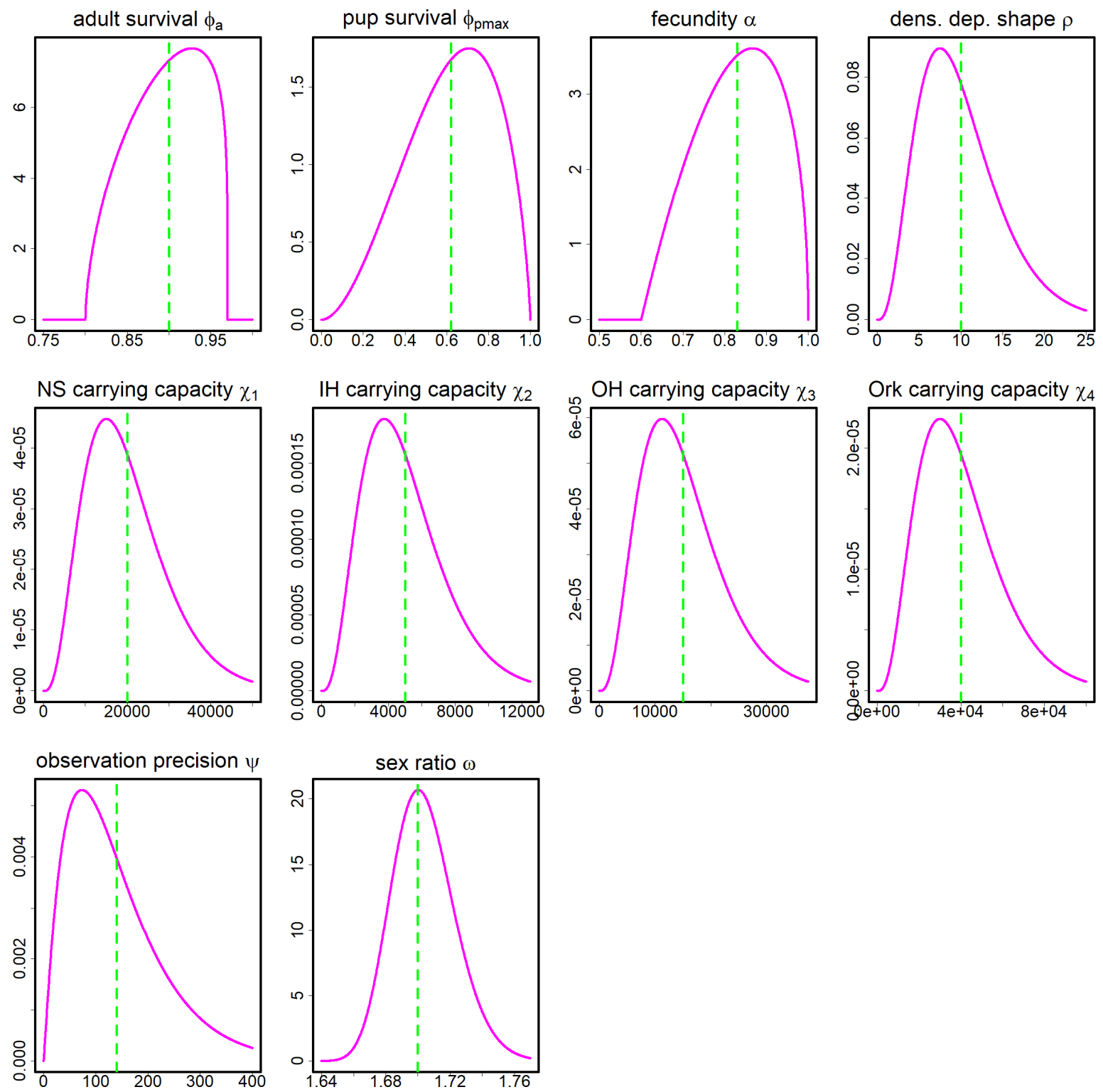

Figure S3. Prior probability density functions for each model parameter, drawn from the distributions specified in Table 1. Prior means are shown as green dashed vertical lines. 
North Sea

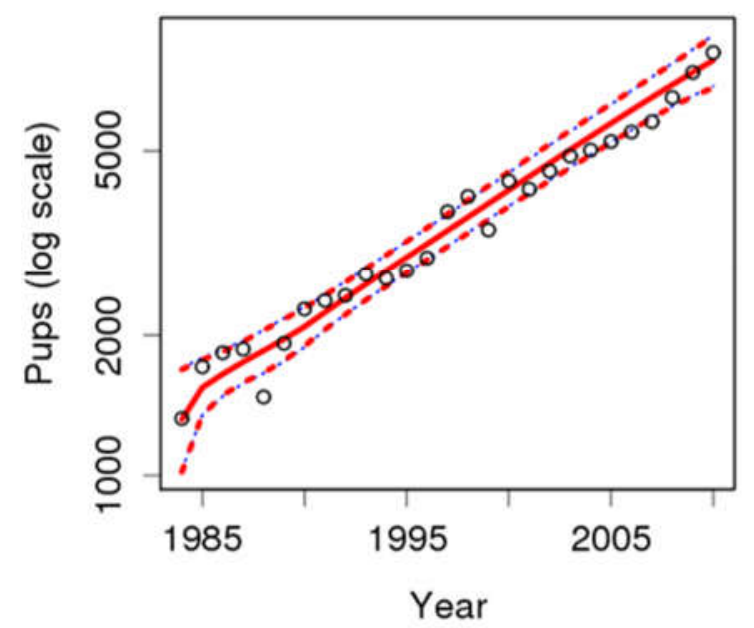

Outer Hebrides

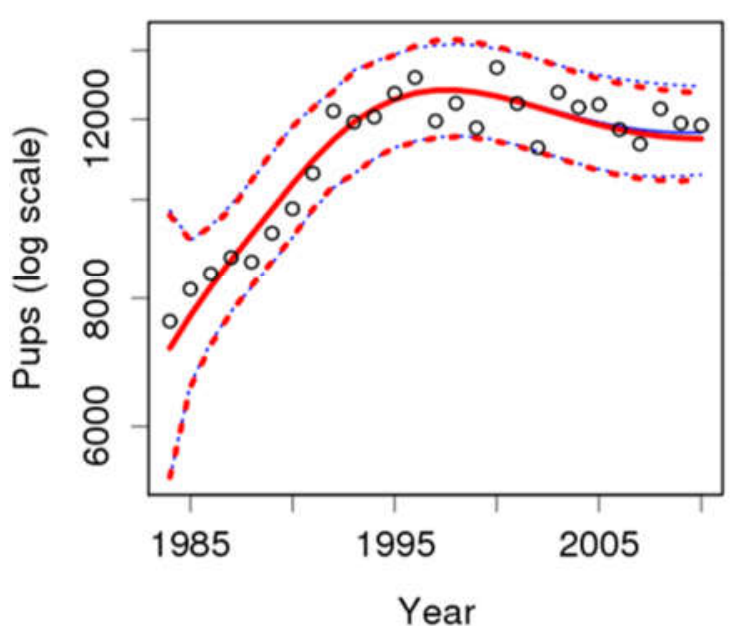

Inner Hebrides

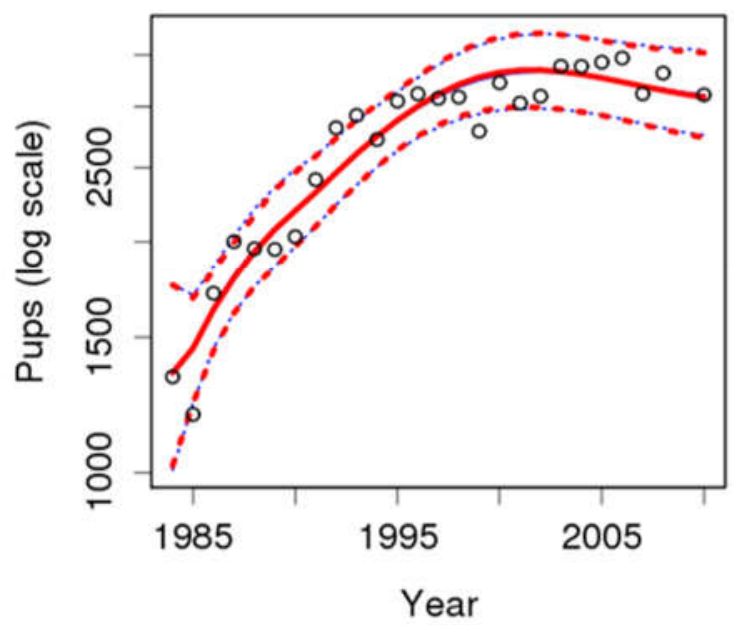

Orkney

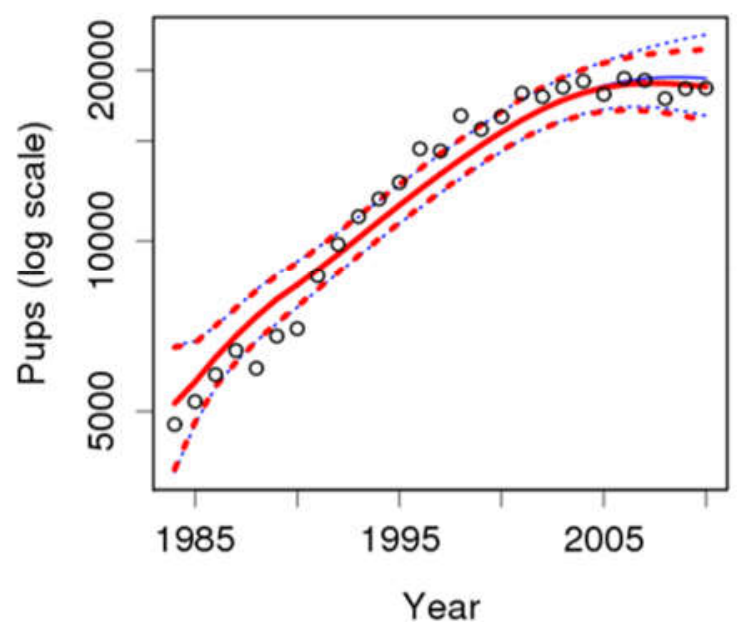

Figure S4. Log-scale plot of posterior mean estimates of regional pup production (solid lines) from the state-space model, with $95 \%$ credible intervals (dashed lines). Thick red lines show results from model fitted to pup production plus the total population estimate of 2008; thinner blue lines show the fit to pup production alone. Circles show pup production data. This is the same information as is shown in Figure 1, but with the $y$-axis on the log scale. On this scale, exponential population growth appears as a straight line. 


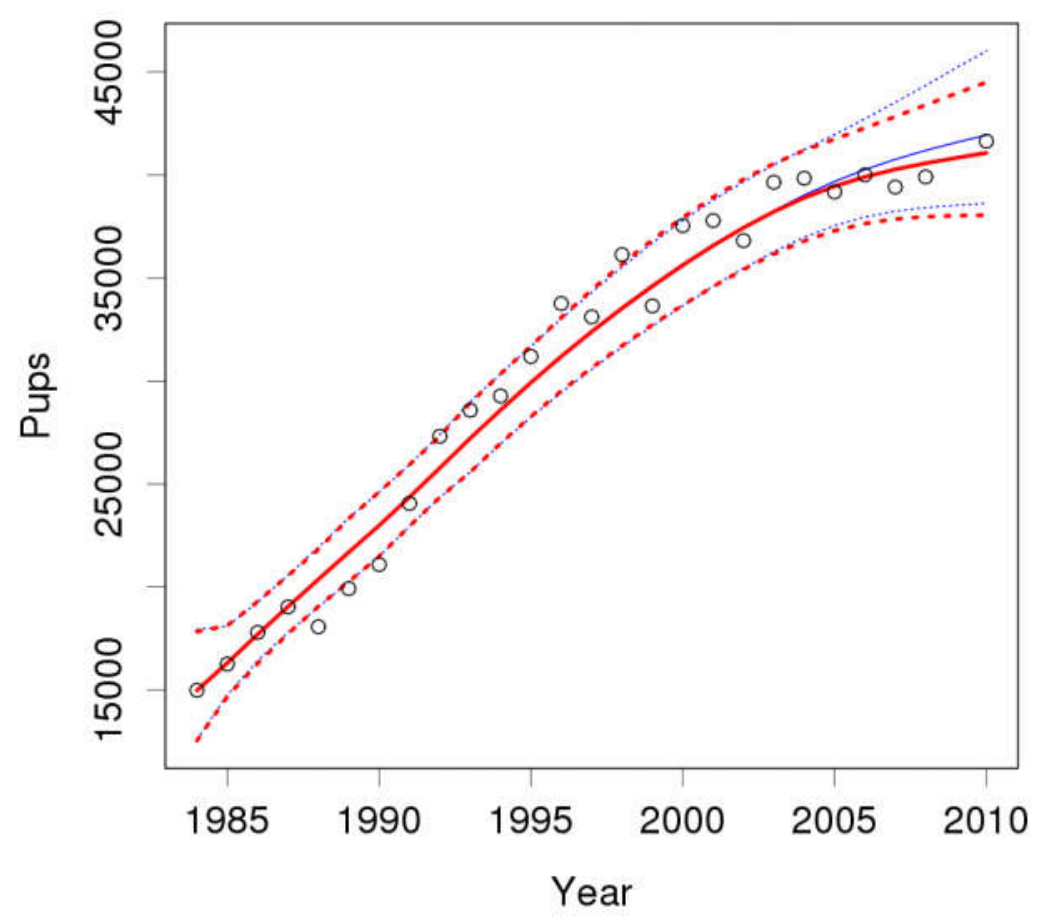

55

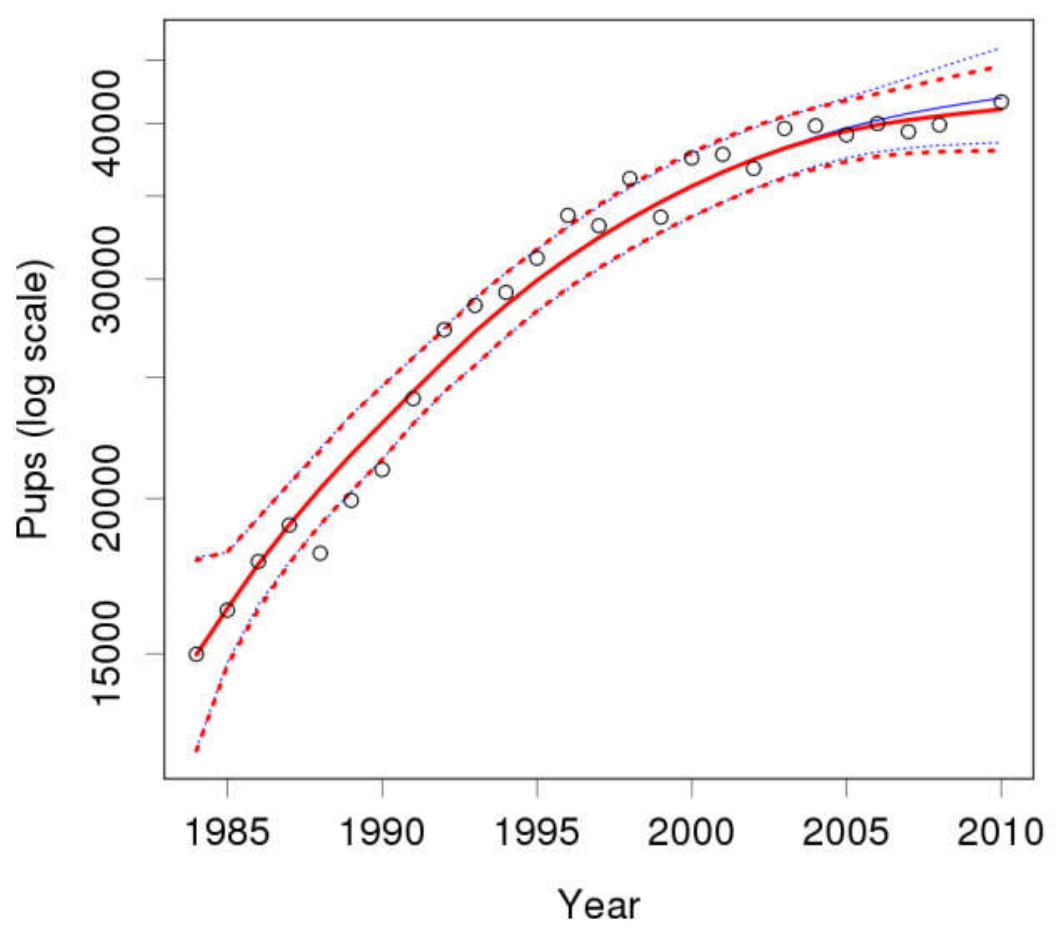

57 Figure S5. Posterior mean estimates of total pup production in annually monitored colonies (solid 58 lines) from the state-space model, with 95\% credible interval (dashed lines). Thick red lines show 59 results from the model the fit to pup production data plus the total population estimate; thinner blue 60 lines show the fit to pup production data alone. Circles show pup production data. The two plots 61 contain the same data, but in the lower one the $y$-axis is on the log scale. 


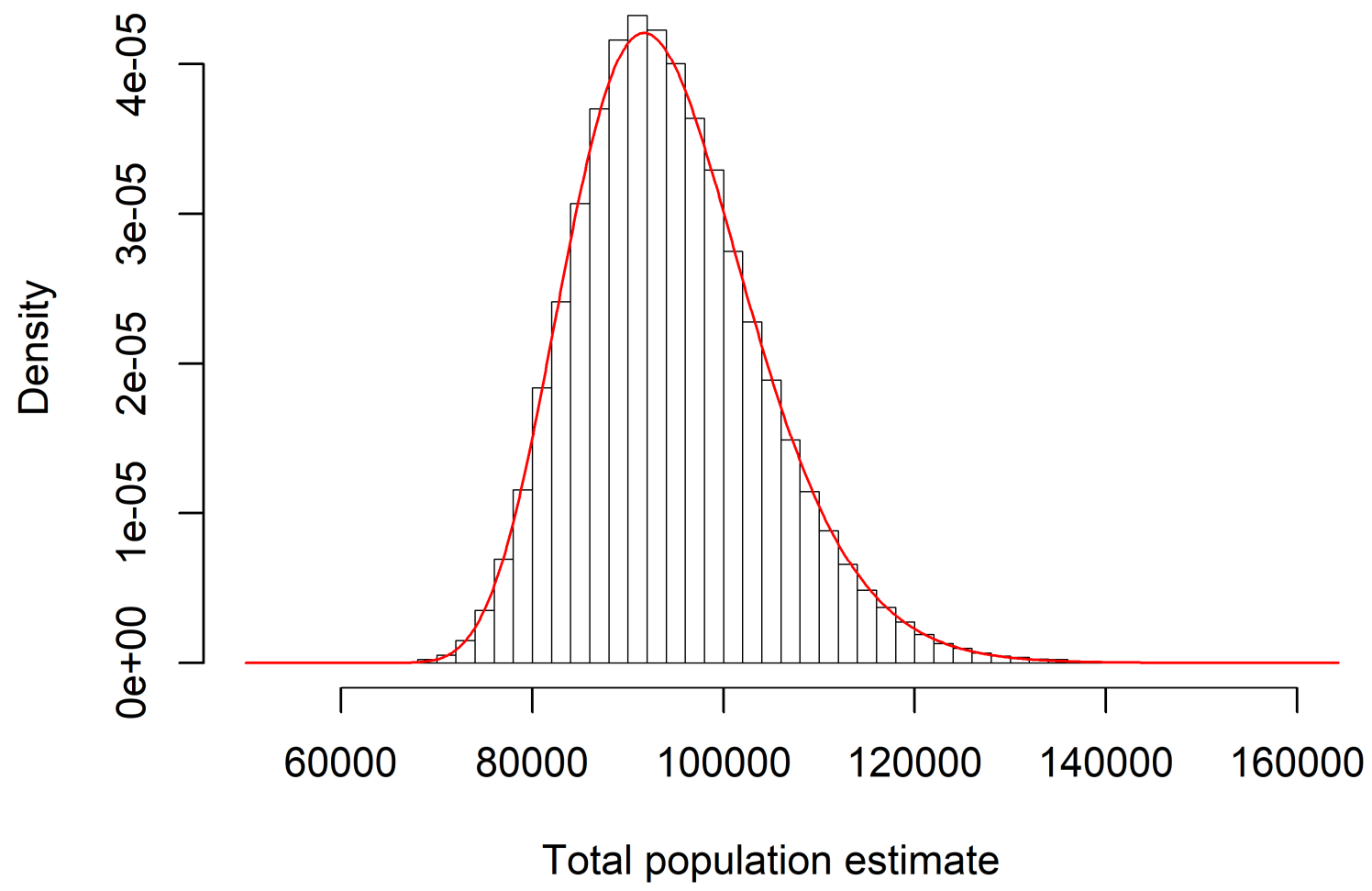

63

64 Figure S6. Histogram showing 100,000 bootstrap estimates of total population size from Russell et al. 65 (2016), after scaling by multiplying by 0.9234 so it represents population size associated with only 66 colonies regularly monitored. Red line shows the fitted right-shifted gamma distribution used in the 67 state-space model, which has parameter values $\kappa_{0}=59167.84161, \kappa_{1}=12.95541, \kappa_{2}=$ $68 \quad 2719.37889$. 


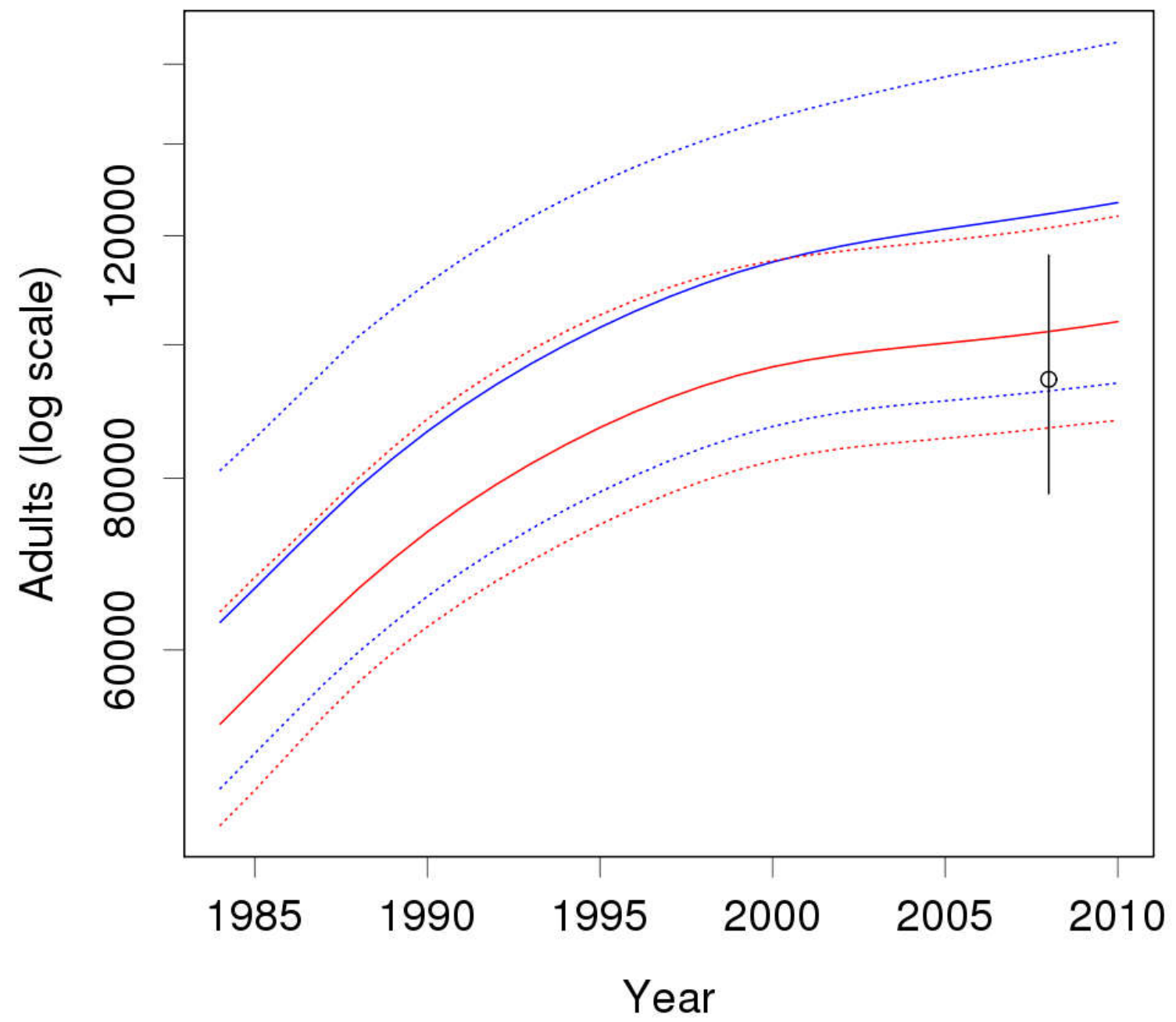

69

70 Figure S7. Log scale plot of posterior mean estimates of adult population size (solid lines) and 95\% 71 credible interval (dashed lines). Thick red lines show the fit to pup production data plus the total 72 population estimate of 2008; thinner blue lines show the fit to pup production data alone. This is the 73 same information as is shown in Figure 2, but with the $y$-axis on the log scale. 
North Sea

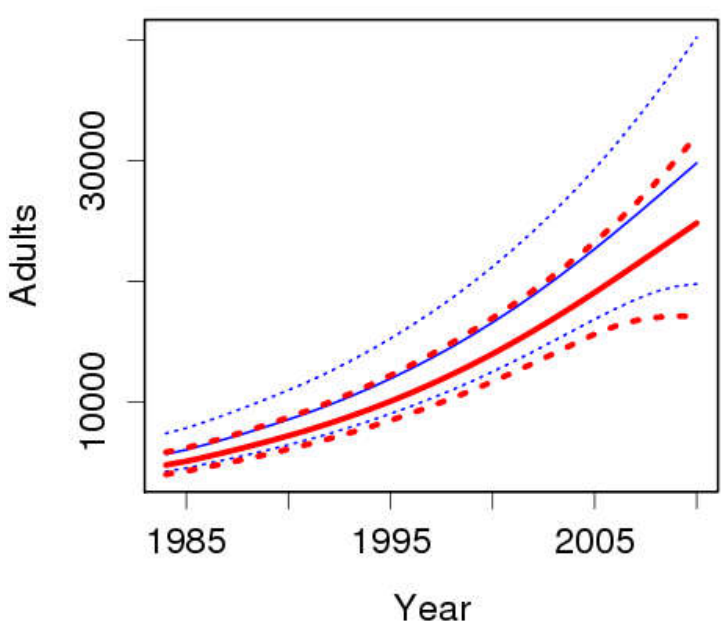

Outer Hebrides

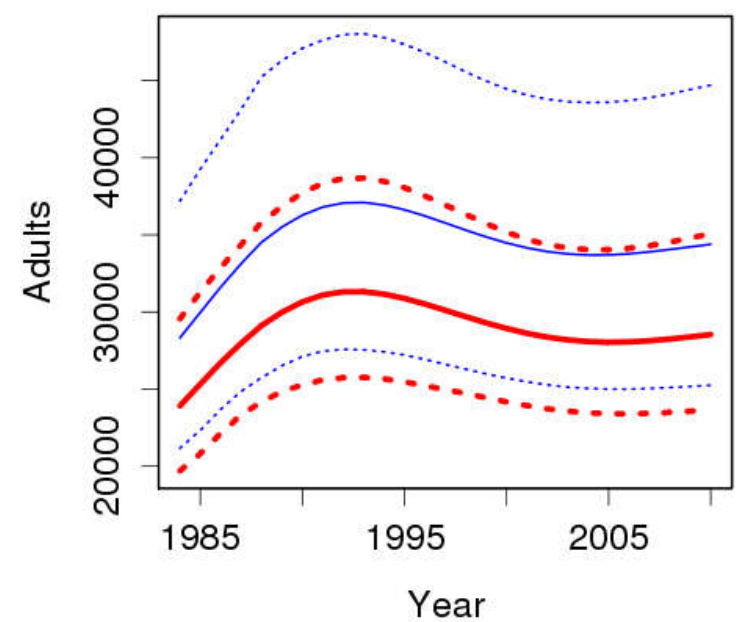

Inner Hebrides

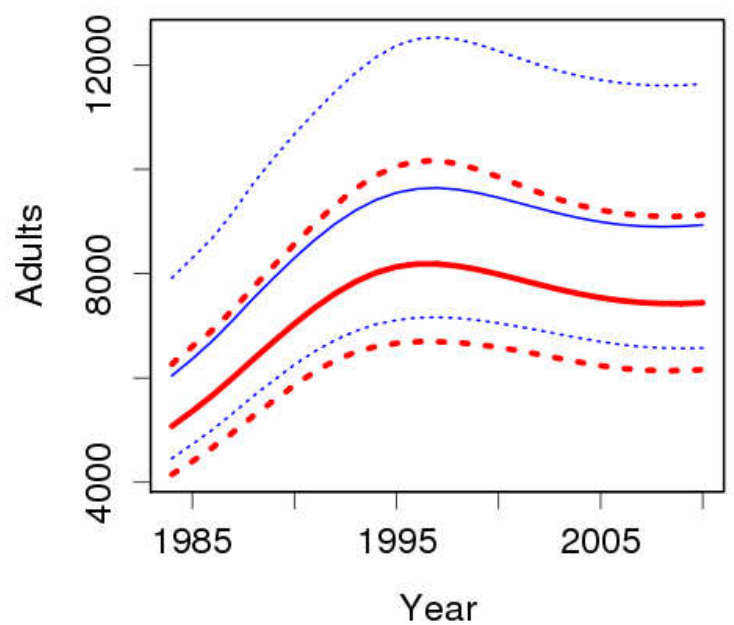

\section{Orkney}

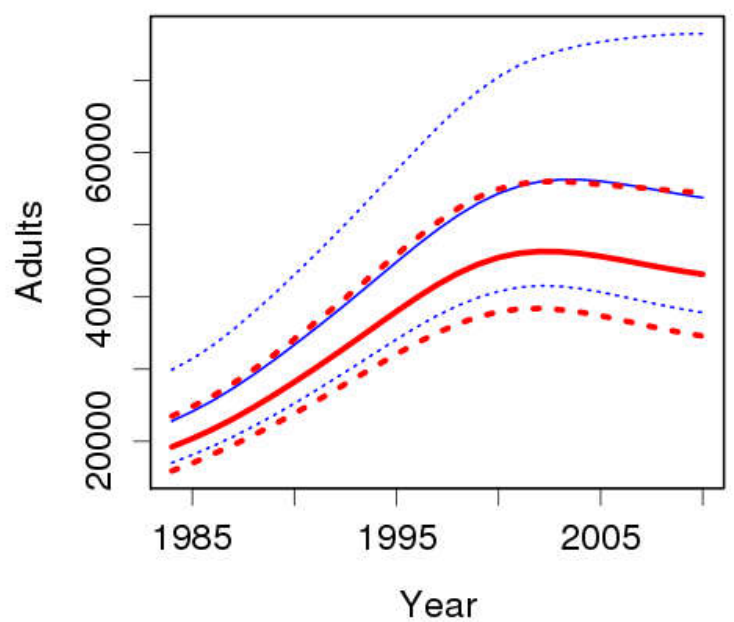

Figure S8. Posterior mean estimates of regional adult population size (solid lines) and $95 \%$ credible interval (dashed lines). Thick red lines show the fit to pup production data plus the total population estimate of 2008; thinner blue lines show the fit to pup production data alone. 


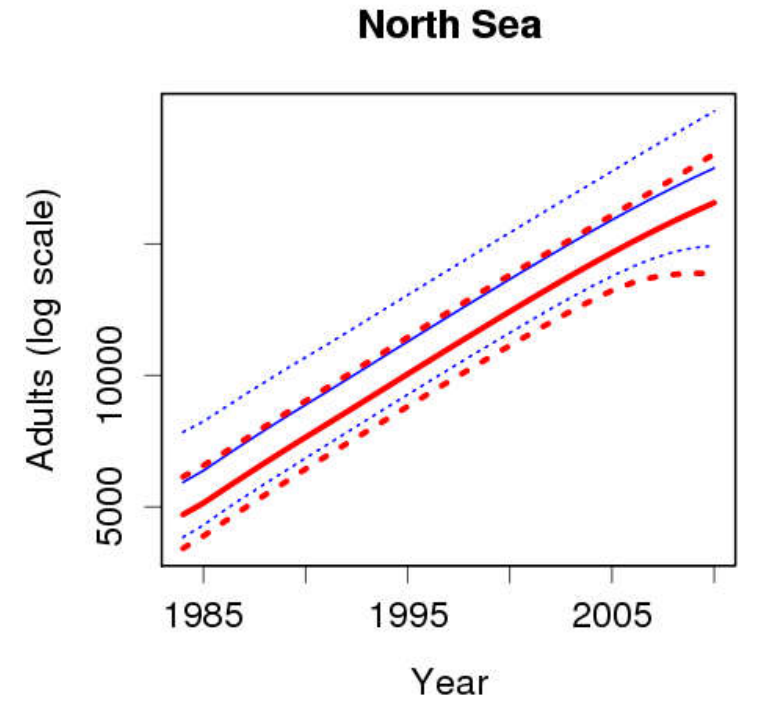

Outer Hebrides

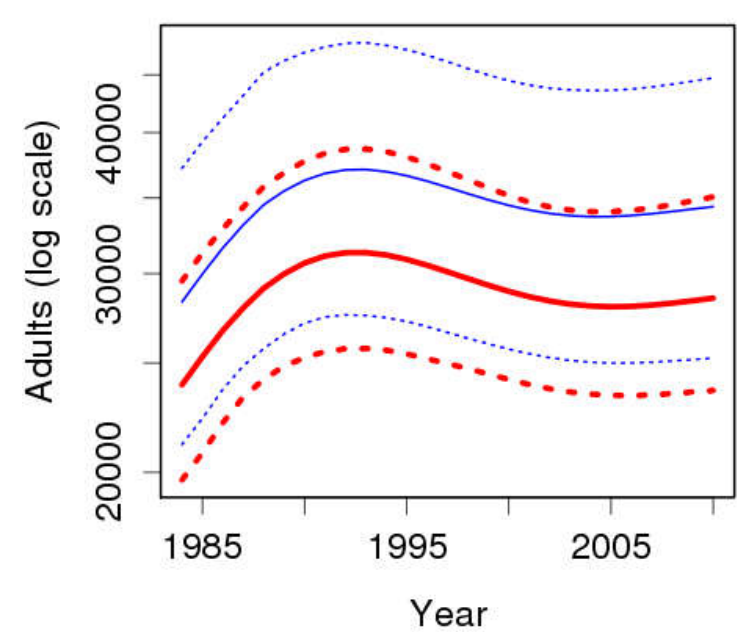

Inner Hebrides

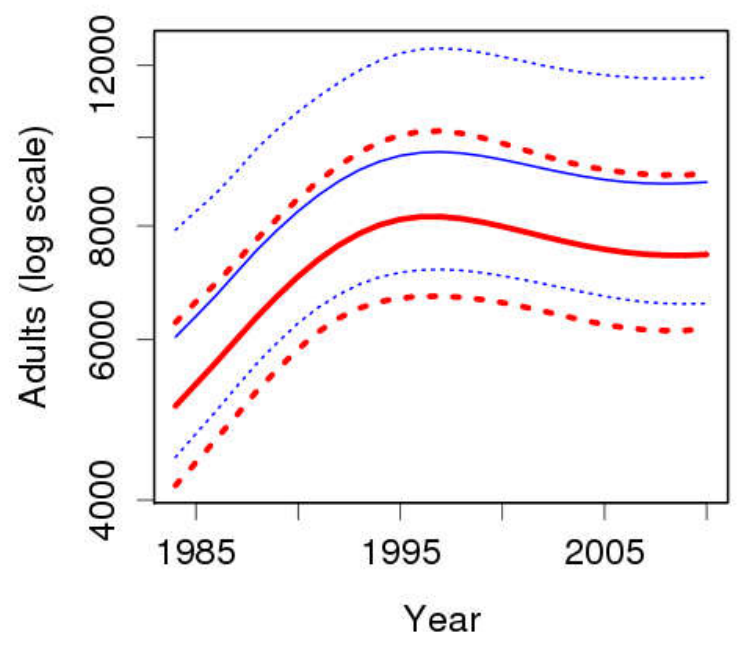

Orkney

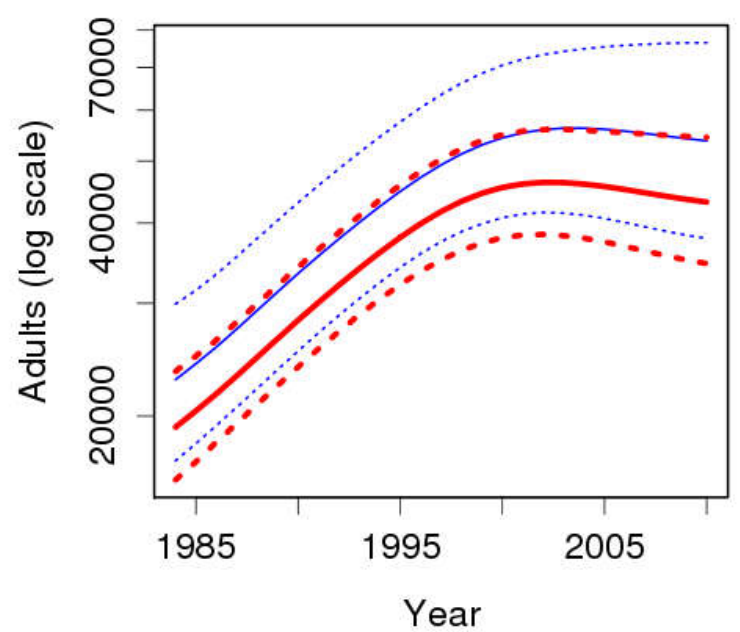

Figure S9. Log-scale plot of posterior mean estimates of regional adult population size (solid lines) and $95 \%$ credible interval (dashed lines). Thick red lines show the fit to pup production data plus the total population estimate of 2008; thinner blue lines show the fit to pup production data alone. This is the same information shown in Figure 58 , but with the $y$-axis on the log scale. 


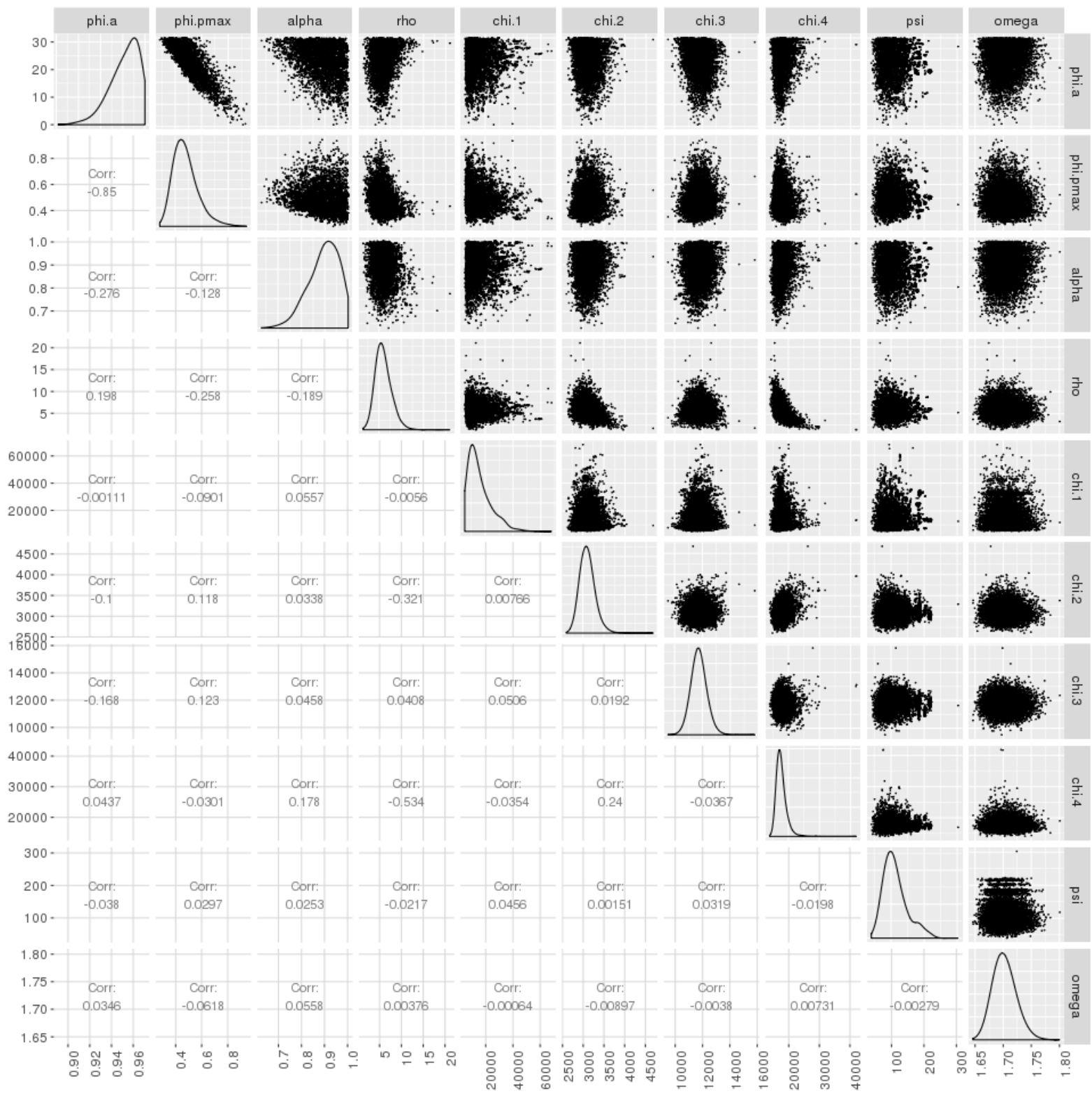

Figure S10. Pairwise scatterplots and correlation coefficients for posterior parameter distributions of the state-space model of grey seal population dynamics, fitted to pup production estimates plus the total population estimate of 2008. Diagonal elements are univariate marginal posterior densities. (Note that, for presentational reasons, a subset of 10,000 posterior samples were selected at random for display.) 

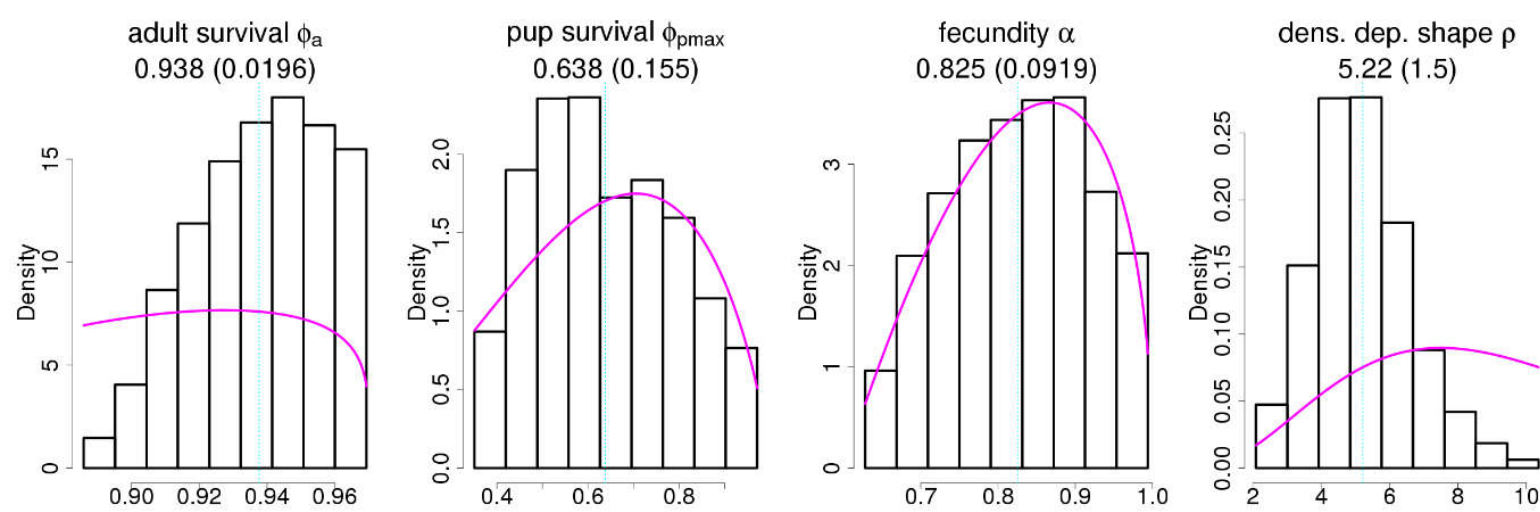

NS carrying capacity $\chi_{1}$ $15900(7870)$
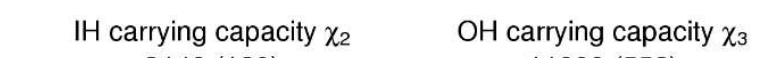

Ork carrying capacity $\chi_{4}$ $3140(180)$
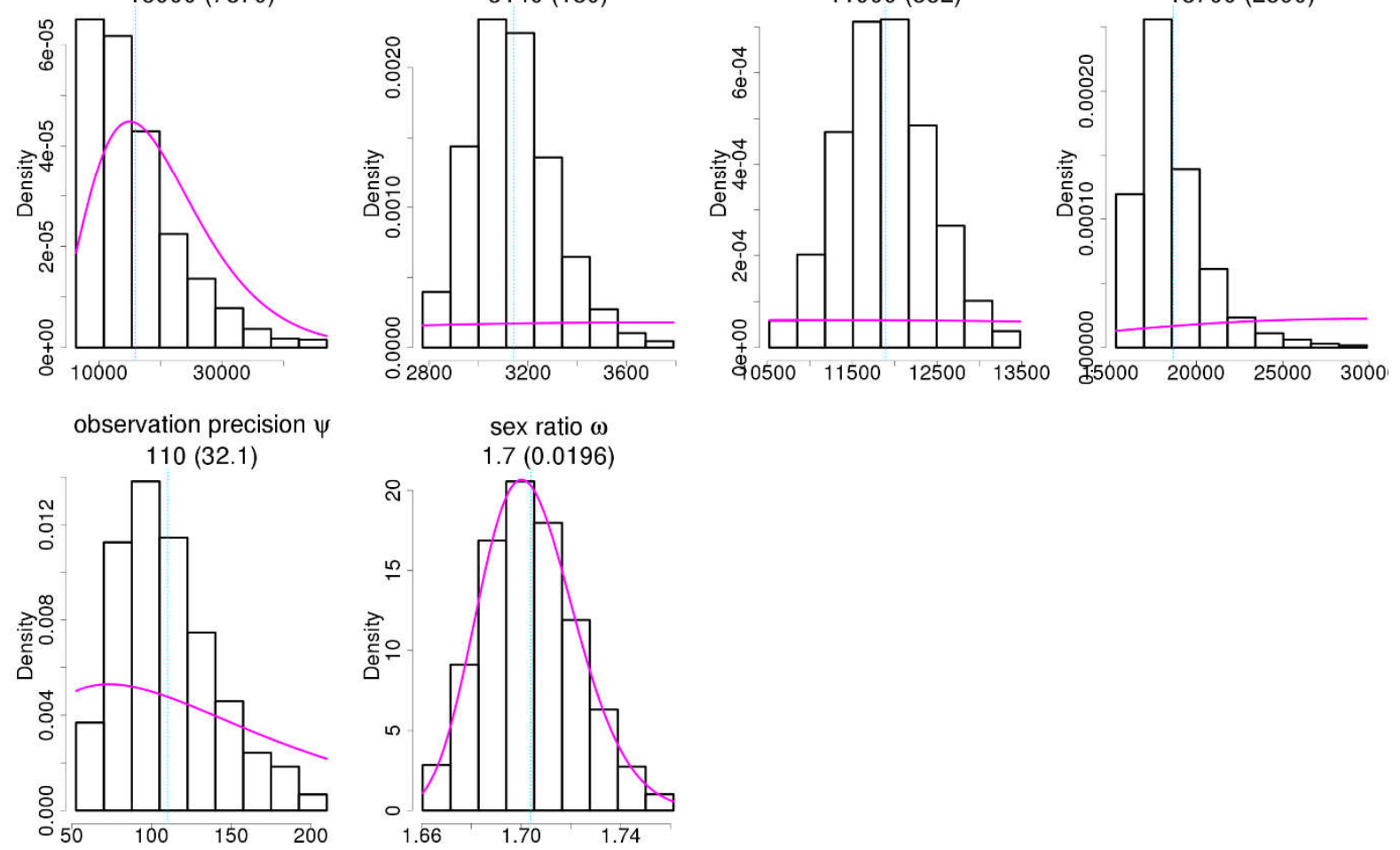

Figure S11. Posterior (histograms) and prior (solid lines) parameter distributions for the state-space model fitted to pup production estimates from 1984-2010 alone - i.e., without the independent estimate of (scaled) total populations size from 2008. The vertical dashed line shows the posterior mean; its value is also given in the title of each plot below the parameter name, with the associated standard deviation in parentheses. 


\section{S2. Justification for prior distributions}

Prior distributions are required for the following model parameters: adult female survival $\phi_{a}$, maximum pup survival $\phi_{p \max }$, fecundity $\alpha$, shape of density dependence acting on pup survival $\rho$, region-specific carrying capacity (in terms of pup production) $\chi_{1-4}$, number of adults per female $\omega$, and precision of the pup production estimates $\psi$. The data used to inform these priors are presented below and in Tables S4 and S5. The resulting prior distributions are summarized in the main paper Table 1 and shown in Figure S3. Further discussion of prior selection is given in Longeran (2012), Longeran (2014) and Russell (2017).

\section{S2.1 Adult female survival $\phi_{a}$}

Relevant studies are summarized in Table S4. Estimates of annual adult survival in the UK, obtained by aging teeth from shot animals are between 0.935 and 0.96 (Harwood \& Prime, 1978; Hewer, 1964; Lonergan, 2012). Capture-mark-recapture (CMR) of adult females on breeding colonies can be used to estimate female survival but may produce underestimates as they are dependent on the assumption that females not returning to the study colony have died. Using capture-mark-recapture (CMR), adult survival was estimated to be 0.886 (95\% Cl 0.871-0.900) and 0.941 (95\% Cl 0.929-0.954) for a declining (North Rona) and increasing (Isle of May) population, respectively (Smout, King \& Pomeroy, submitted). Based on the above data, and the fact that the lower limit on adult survival cannot be lower than 0.8 (Lonergan, 2012), the prior on adult female survival is specified as a beta distribution $\mathrm{Be}(1.6,1.2)$ which is scaled (multiplied by 0.17 and added to 0.8 ) to allow non-zero probability density only between 0.8 and 0.97 . The resulting distribution has mean 0.90 and SD 0.04 . However, recent estimates from Sable Island suggest adult female survival may be above this upper bound. den Heyer \& Bowen (2017) used a Cormack-Jolly-Seber model to estimate age- and sex-specific adult survival from a long-term brand re-sighting programme on Sable Island. Average female adult survival was estimated to be 0.976 (SE 0.001), averaged over all animals, but was higher for younger adults (0.989 with SE 0.001 for age classes 4-24) than older adults (0.904 SE 0.004 for age 25+).

\section{S2.2 Maximum pup survival $\phi_{\text {pmax }}$}

Relevant studies are summarized in Table S4. Data from populations that were growing rapidly and therefore apparently not constrained by density dependence acting on pup survival were required to inform this prior. There are various published estimates of first-year survival during periods of exponential growth (Table S4). Mean estimates of pup surival were between $0.54-0.76$. On the basis of these estimates, the prior on maximum female pup survival is defined as a diffuse beta distribution $\mathrm{Be}(2.87,1.78$ ) which has mean of 0.62 (SD 0.20). Note that Pomeroy, Smout, Moss, Twiss, \& King (2010) found high inter-annual variation in pup survival, which is not currently incorporated in the model.

\section{S2.3 Fecundity $\alpha$}

Relevant studies are summarized in Table S5. For the purposes of this model, fecundity refers to the proportion of breeding-age females (aged 6 and over) that give birth to a pup in a year (natality or birth rate). For the most part, studies have measured pregnancy rather than natality rates. The resulting estimates are thus maxima in terms of fecundity as abortions will cause pregnancy rates to exceed birth rates. Mean estimated adult female pregnancy rates from examination of shot animals were between 0.83 and 0.94 in the UK (Boyd, 1985; Hewer, 1964), and between 0.88 and 1 at Sable Island, Canada (Hammill \& Gosselin, 1995). CMR studies report lower estimates, which may be a result of unobserved pupping events (due to mark misidentification, tag loss, or breeding elsewhere), but also because such estimates represent births rather than pregnancy. Such studies, from Sable Island estimate fecundity to be between 0.57 and 0.83 (Bowen, Iverson, McMillan, \& Boness, 2006; den Heyer \& Bowen, 2017). UK estimates of fecundity rates for populations of marked study animals, adjusted for estimates of unobserved pupping events were 0.790 (95\% Cl 0.766-0.812) and 0.816 (95\% $\mathrm{Cl}$ 0.787-0.841) for a declining (North Rona) and increasing (Isle of May) population, respectively (Smout et al., Submitted). Based on the available data, the prior on fecundity $(\alpha)$ is specified as a 
beta distribution $\mathrm{Be}(2,1.5)$ which is scaled (multiplied by 0.4 and added to 0.6 ) to only allow probability density between 0.6 and 1 . The resulting distribution has mean 0.83 and SD 0.09 .

\section{S2.4 Shape of density dependence acting on pup survival $\rho$}

Pup survival at carrying capacity is not dependent on this parameter (Eqn. 4, main paper), and hence carrying capacity also does not depend on it. Instead, the parameter influences the shape of the population growth trajectory, by determining the shape of the relationship between pup survival and pup production (Figure S1). Fowler (1981) used both theory and empirical data to suggest that most density-dependent change in vital rates happens close to carrying capacity for species with life history strategy typical of large mammals (i.e., long lived and low reproductive rate). Empirical examples (their Figure 4) show relationships consistent with values of $\rho$ in the range 5-10. To avoid being too prescriptive, a diffuse distribution was specified: a Gamma distribution $\mathrm{Ga}(4,2.5)$, which has a mean of 10 and SD 5.

\section{S2.5 Region-specific pup production at carrying capacity $\chi_{1} 4$}

No independent information was available about carrying capacity, and so the priors were specified with a variance wide enough to make their influence on population size estimates negligible. Truly non-informative priors (e.g., improper priors with infinite variance) make the particle filtering algorithm extremely inefficient, since most simulated trajectories are infeasible given the data, hence a trade-off is required between a prior with a large enough variance to be non-informative, but not too large so as to make the algorithm prohibitively inefficient. Having the initial rejection control step in the algorithm helped to some extent in this regard. Gamma distributions with a SD:mean ratio of 1:2, with the mean set subjectively based on expert opinion (Table 1) were found to meet these criteria. For the three regions where pup production increased to an asymptote (Inner Hebrides, Outer Hebrides and Orkney) the posterior overwhelmed the prior (prior-posterior overlap $<15 \%$, Table 1). For the remaining region (North Sea), where pup production is still growing at a nearexponential rate, the estimate of population size is not strongly affected by density dependent processes and any prior distribution that is reasonably far above the current population counts will have no influence on historical or current estimates of population size in that region. Note, however, that since there is little information with which to set this prior, one should be cautious with interpretation regarding carrying capacity in the case (as with North Sea) that the prior strongly influences the posterior (see also Section 4.2, main paper).

\section{S2.6 Number of adults per female $\omega$}

This parameter is also referred to as the sex ratio, although strictly the ratio of males:females is given by $\omega \quad 1$. Relevant studies (on sex-specific survival rates) are summarized in Table S4. A sex ratio of 0.73:1 was derived from shot samples (Harwood \& Prime, 1978). This was based on the following assumptions: that the shot males were a representative sample of the breeding population ( $\geq 10$ years old); that female survival was 0.935; and that survival was the same between the sexes up until age 10. Using telemetry tags and "hat tag" re-sighting data (taking into account detection probability inferred by telemetry data), sex-specific pup survival was estimated (Lonergan 2014; Table A1). Although there were no significant differences in survival between males and females, the mean male survival was lower than females. Combined with data from Hewer (1964), the resulting sex ratio would be between 0.66:1 and 0.68:1 (Lonergan, 2014). Also considered were pup survival estimates derived from shot samples from the Baltic (Kauhala, Ahola, \& Kunnasranta, 2012). For Sable Island, the sex ratio is estimated to be 0.69:1 based on estimates of age and sex-specific survival, and assuming a stationary age distribution (Hammill, den Heyer, Bowen, \& Lang, 2017). Based on these findings, the prior used was a highly informative scaled Gamma distribution $\mathrm{Ga}(4,2.5)+1.6$. This results in a prior mean of 1.7 (SD 0.02); $90 \%$ of the prior probability density is between 1.68 and 1.73 . 


\section{S2.6 Precision of the pup production estimates $\psi$}

The pup production estimates at colony level from aerial survey data generally have a coefficient of variation of $10 \%$ or less (Russell et al. submitted). Uncertainty in the ground count estimates is not quantified. The resulting uncertainty in pup production at the region level is hard to predict - if the colony estimates were independent it would be smaller, but they are not independent since they share some parameters (Russell et al. submitted). Hence a moderately diffuse prior was specified on $\psi$ $(\mathrm{Ga}(2.1,66.67)$, implying a prior on $\mathrm{CV}$ of pup production (which is $1 / \psi)$ of $10 \%$ with SD 5 (i.e., with $90 \%$ of the prior probability density between $5 \%$ and $20 \%$ ).

\section{S3. Details of state-space model fitting algorithm}

Note that computer code to implement this algorithm for the grey seal state-space model, in ANSI C, is provided as further Supporting Information.

Let $\theta$ denote the vector of model parameters, $p(\theta)$ the prior probability density function (pdf) of these parameters, $n_{t}$ the vector of states at time $t$ (length 28 ( 7 ages $\times 4$ regions)), $g_{0}\left(n_{0} \mid \theta\right)$ the prior pdf of the states at time $\mathrm{t}=0, g\left(n_{t} \mid n_{t-1}, \theta\right)$ the state process pdf, $y_{t}$ the vector of pup production observations at time $t$ ( 4 regions) and $f\left(y_{t} \mid n_{t}, \theta\right.$ ) be the observation process pdf. $t=0$ corresponds to 1984 , while $\mathrm{t}=26$ corresponds to 2010 ; the symbol $T$ is also used for the last time period. Let $p\left(n_{1: T}, \theta \mid y_{1: T}\right)$ denote the posterior distribution of the states and parameters given the observations.

The main algorithm, to generate a weighted set of $N$ particles drawn from $p\left(n_{1: T}, \theta \mid y_{1: T}\right)$ is as follows.

1. Initialize. Generate $N$ draws of $\theta$ and $n_{0}$ ( "particles") by sampling from $p(\theta)$ and $g_{0}(\theta)$. Denote these $\theta^{[i]}$ and $n_{0}^{[i]}$ where $i=1, \ldots, N$.

2. Initial rejection control.

a. For each particle, generate a value for $n_{1}^{[i]}$ from $g\left(n_{1} \mid n_{0}^{[i]}, \theta^{[i]}\right)$ (i.e., simulate one time step forward using the population dynamics model) and calculate the likelihood weights $w_{1}{ }^{[i]}=f\left(y_{1} \mid n_{1}^{[i]}, \theta^{[i]}\right)$.

b. Retain each particle with probability equal to $r_{1}^{[i]}=\min \left(w_{1}{ }^{[i]} / w_{c}, 1\right)$ where $w_{c}=$ $\frac{1}{N} \sum_{j=1}^{N} w_{1}{ }^{[j]}$ is the mean of the weights. For those particles retained, set $w_{1}^{[i]}=$ $w_{1}{ }^{[i]} / r_{1}^{[i]}$. Standardize the weights so they have a mean (over all particles) of 1 (see below).

3. Repeat steps 1 and 2 until at least $N$ particles have been retained (if more than $N$ are retained then keep only the first $N$ ). Set $t=1$.

4. Auxiliary particle filter with kernel smoothing.

a. Set $t=t+1$

b. For each particle, calculate the expected value $n_{t}{ }^{[i]}=E\left(n_{t} \mid n_{t-1}^{[i]}, \theta^{[i]}\right)$. (This is simply a deterministic projection one time step forward using the population dynamics model.) Calculate the likelihood weights $w_{t}{ }^{[i]}=w_{t-1}^{[i]} f\left(y_{t} \mid n_{t}{ }^{[i]}, \theta^{[i]}\right)$.

c. Resample $N$ particles with replacement using tempered resampling to retain particle diversity, with normalized probability weights $q_{t}^{[i]} \propto\left(w_{t}{ }^{[i]}\right)$. A residual resampling algorithm (Lui and Chen 1998) is used to reduce Monte Carlo error:

i. For $i=1, \ldots, N$, keep $k_{t}^{[i]}=\left|N q_{t}^{[i]}\right|$ copies of particle $i$.

ii. Set $\widetilde{N}=N \quad \sum_{\{i=1\}}^{N} k_{t}^{[i]}$ and $\tilde{q}_{t}^{[i]} \propto N q_{t}^{[i]} \quad k_{t}^{[i]}$ for $i=1, \ldots, N$.

iii. Sample $\widetilde{N}$ particles with replacement with probabilities $\tilde{q}_{t}^{[i]}$.

d. Using the resampled set of particles, kernel smooth the parameters. 
i. Transform $(0,1)$ bounded parameters with a logit transform; transform $[0, \infty)$ bounded parameters with a log transform.

ii. Generate a new parameter vector $\theta^{[i]}=\lambda \theta^{[i]}+\left(\begin{array}{ll}1 & \lambda\end{array}\right) Z_{i}$ where $\lambda$ is a tuning parameter with $0 \leq \lambda \leq 1$ (see below) and $Z_{i}$ is a sample from a multivariate normal distribution with mean vector $\bar{\theta}=\sum_{i=1}^{n} w_{i} \theta^{[i]} / \sum_{i=1}^{n} w_{i}$ and covariance matrix made up of the variances and covariances of the $\mathrm{N}$ vectors $\theta^{[i]}$. The tuning parameter $\lambda$ controls the degree of smoothing; in the runs reported here, minimal smoothing was applied by setting $\lambda=0.99997$.

iii. Set $\theta^{[i]}=\theta^{[i]}$

e. For each resampled particle, generate a value for $n_{t}^{[i]}$ from $g\left(n_{t} \mid n_{t-1}^{[i]}, \theta^{[i]}\right)$ and calculate the weights $w_{t}^{[i]}=w_{t-1}^{[i]} f\left(y_{t} \mid n_{t}^{[i]}, \theta^{[i]}\right) / q_{t}^{[i]}$. Standardize the weights so they have a mean (over all particles) of 1 (see below).

5. Repeat step 4 until $t=T$.

6. Final rejection control. Standardize the weights so they have a mean (over all particles) of 1 (see below) and call them $w_{T}{ }^{[i]}$. Retain each particle with probability equal to $\min \left(w_{T}^{[i]} / w_{c}, 1\right)$ where $w_{c}=100$. For those particles retained, set $w_{T}^{[i]}=w_{T}{ }^{[i]} / r_{T}^{[i]}$.

The result is a set of weighted particles $\left\{\left(n_{1: T}^{[i]}, \theta^{[i]}, w_{T}^{[i]}\right)\right\}, i=1, \ldots, N$ that represent a weighted sample from the posterior $p\left(n_{1: T}, \theta \mid y_{1: T}\right)$. To make inferences about state or parameter values, appropriate weighted averages can be calculated - for example the posterior mean estimate of the parameters is given by $\hat{E}\left(p\left(\theta \mid y_{1: T}\right)\right)=\sum_{i=1}^{N} w_{T}^{[i]} \theta^{[i]} / \sum_{i=1}^{N} w_{T}^{[i]}$.

In practice, to provide sufficient Monte Carlo accuracy the above algorithm was executed 400 times, each time with $\mathrm{N}=1,000,000$, and the resulting particles combined (after a further rejection control step with $\left.w_{c}=1,000\right)$. A step that has not been documented here is standardizing the weights: at each time step the weights were standardized so their mean was approximately 1 , in order to prevent numerical underflow. The standardization must use the same divisor in each of the 400 iterations, and so the divisors were pre-calculated in an initial pilot run of the algorithm.

\section{References}

Bowen, W. D., Iverson, S. J., McMillan, J. I., \& Boness, D. J. (2006). Reproductive performance in grey seals: age-related improvement and senescence in a capital breeder. Journal of Animal Ecology, 75(6), 1340-1351. http://doi.org/10.1111/j.1365-2656.2006.01157.x

Boyd, I. (1985). Pregnancy and ovulation rates in grey seals (Halichoerus-grypus) on the British coast. Journal of Zoology, 205, 265-272.

Carter, M. I. D., Russell, D. J. F., Embling, C. B., Blight, C. J., Thompson, D., Hosegood, P. J., \& Bennett, K. A. (2017). Intrinsic and extrinsic factors drive ontogeny of early-life at-sea behaviour in a marine top predator. Scientific Reports, 7(1), 1-14. http://doi.org/10.1038/s41598-017-15859-8

den Heyer, C. E., \& Bowen, W. D. (2017). Estimating changes in vital rates of Sable Island grey seals using mark-recapture analysis. DFO Can. Sci. Advis. Sec. Res. Doc. 2017/054., 27.

den Heyer, C. E., Bowen, W. D., \& Mcmillan, J. I. (2014). Long-term Changes in Grey Seal Vital Rates at Sable Island Estimated from POPAN Mark-resighting Analysis of Branded Seals. DFO Can. Sci. Advis. Sec. Res. Doc. 2013/021, (April), 26.

Fowler, C.W. 1981. Density dependence as related to life history strategy. Ecology 62: 602-610.

Hall, A. J., McConnell, B. J., \& Barker, R. J. (2001). Factors affecting first-year survival in grey seals and their implications for life history strategy. Journal of Animal Ecology. 
Hall, A. J., Thomas, G. O., \& McConnell, B. J. (2009). Exposure to persistent organic pollutants and firstyear survival probablility in gray seal pups. Environmental Science \& Technology, 43(16), 63656369.

Hall, A., McConnell, B., \& Barker, R. (2002). The effect of total immunoglobulin levels, mass and of Grey Seal pups condition on the first-year survival, 16(4), 462-474.

Hammill, M. O., den Heyer, C. E., Bowen, W. D., \& Lang, S. L. C. (2017). Grey Seal Population Trends in Canadian Waters, 1960-2016 and harvest advice. Research Document , (October), 1-35.

Hammill, M. O., \& Gosselin, J. (1995). Grey seal (Halichoerus grypus) from the Northwest Atlantic: Female reproductive rates, age at first birth, and age of maturity in males. Canadian Journal of Fisheries and Aquatic Sciences, 52(12), 2757-2761.

Harwood, J., \& Prime, J. H. (1978). Some factors affecting size of British grey seal populations. Journal of Applied Ecology, 15(2), 401-411. http://doi.org/10.2307/2402600

Hewer, H. (1964). The determination of age, in the grey seal (Halichoerus grypus) sexual maturity, longevity and a life-table. Proceedings of The Zoological Society of London, 142(4), 593-623.

Hewer, H. (1974). British seals. New York: Taplinger Publishing Co. Inc.

Kauhala, K., Ahola, M., \& Kunnasranta, M. (2012). Demographic structure and mortality rate of a Baltic grey seal population at different stages of population change, judged on the basis of the hunting bag in Finland. Annales Zoologici Fennici, 49, 287-305.

Lonergan, M. (2012). Priors for grey seal population model. SCOS Briefing paper 12/02, Sea Mammal Research Unit, University of St Andrews. http://www.smru.st-andrews.ac.uk/researchpolicy/scos/

Lonergan, M. (2014). Addendum to: Lonergan, M. 2013. The case for moving away from 73 males per 100 female. SCOS Briefing paper 14/04, Sea Mammal Research Unit, University of St Andrews. http://www.smru.st-andrews.ac.uk/research-policy/scos/

Lui, J.S. \& Chen, R. (1998) Sequential Monte Carlo methods for dynamics systems. Journal of the American Statistical Association 93, 1032-1044.

Pomeroy, P. P., Smout, S., Moss, S., Twiss, S. D., \& King, R. (2010). Low and Delayed Recruitment at Two Grey Seal Breeding Colonies in the UK. Journal of Northwest Atlantic Fishery Science, 42, 125-133. http://doi.org/10.2960/J.42.m651

Russell, D. 2017 Annual review of priors for grey seal population model. SCOS Briefing paper 17/01A, Sea Mammal Research Unit, University of St Andrews. http://www.smru.standrews.ac.uk/research-policy/scos/

Schwarz, C. J., \& Stobo, W. T. (2000). Estimation of juvenile survival, adult survival, and age-specific pupping probabilities for the female grey seal (Halichoerus gryprus) on Sable Island from capture - recapture data, 253(1994), 247-253.

Smout, S., King, R., \& Pomeroy, P. (Submitted). Environment-sensitive mass changes influence breeding in a marine top predator. Journal of Animal Ecology. 